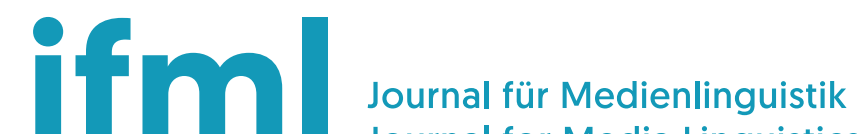 \\ Journal for Media Linguistics
}

Vol 1 (2018), No 1: $42-79$

DOI: $10.21248 / \mathrm{jfml} .2018 .6$

Gutachten und Kommentare unter:

http://dp.jfml.org/2018/06/19/opr-mostovaia-graphische-ressourcen-inder-interaktionalen-informellen-schriftlichkeit

\section{Nonverbale graphische Ressourcen bei Reparaturen in der interaktionalen informellen Schriftlichkeit am Beispiel der deutschen Chat-Kommunikation via IRC- Chat und WhatsApp}

Irina Mostovaia

\begin{abstract}
The aim of this paper is to present the results of an empirical analysis of the use of non-alphabetic graphic signs (e.g. asterisks, slashes, plus signs etc.) in the context of repairs in Russian and German informal electronic communication. The data for the analysis were taken from the „Mobile Communication Database MoCoDa“ (http:// mocoda.spracheinteraktion.de/), which contains Russian and German private electronic communication via SMS, WhatsApp and other short message services, and the „Dortmunder Chat-Korpus“ (http://www.chatkorpus.tu-dortmund.de/korpora.html). This paper describes the functions of various graphic resources in the context of repairs in both data collections and compares the occurrences of these functions in current Russian and German computer-mediated communication. It concludes that particular signs in both datasets share the same subset of functions, but they differ in terms of how frequently these resources occur in each form of communication.
\end{abstract}

Keywords: repair, repair initiation, WhatsApp, Internet Relay Chat (IRC), computer-mediated communication (CMC), asterisk, equal sign, plus sign, minus sign 


\section{Einleitung}

In der interaktionalen informellen Schriftlichkeit kommen wie in der gesprochenen Sprache unterschiedliche (potentielle) Probleme bei der Sprachproduktion und -rezeption sowie dem Verstehen (vgl. Schegloff et al. 1977) vor, die mit Hilfe von Reparaturen gelöst werden können (vgl. Collister 2011; Jacobs/Garcia 2013; Meredith/Stokoe 2013). Auf die Anwendung des ursprünglich für die gesprochene Sprache entwickelten Konzepts der Reparaturen auf die Analyse interaktionaler schriftlicher Daten wurde bereits in einer Reihe einschlägiger Untersuchungen ausführlich eingegangen (vgl. Jacobs/ Garcia 2013; Meredith/Stokoe 2013; Schönfeldt/Golato 2003), sodass in der vorliegenden Arbeit eine solche Diskussion aus Platzgründen ausgeklammert wird.

Reparaturen in der schriftbasierten Kommunikation weisen sowohl Gemeinsamkeiten als auch Unterschiede zu denen in der gesprochenen Sprache auf. Bei Reparaturinitiierungen können SchreiberInnen beispielsweise zum einen auf verbale Mittel zurückgreifen, die ihnen aus der gesprochenen Sprache vertraut sind, wie z. B. die Partikel $\ddot{a} h$ oder den Diskursmarker ${ }^{1}$ also (vgl. Mostovaia i. V.). Zum anderen nutzen SchreiberInnen kreativ die Möglichkeiten der medialen Schriftlichkeit, indem sie u. a. Fragezeichen, Asteriske oder die Farbmarkierung eines Textausschnittes bei der Reparaturinitiierung und/oder -durchführung einsetzen. Die aus der gesprochenen Sprache (angepasst) übertragenen Ressourcen für die Reparaturinitiierung sind bislang systematisch nur in Ansätzen erforscht (vgl. Mostovaia i. V.). Der Fokus der vorliegenden Arbeit liegt auf denjenigen Initiierungsmitteln, die nur in der (zumindest teilweise) medial schriftlichen computervermittelten Kommunikation (CMC) möglich sind, nicht aber in der mündlichen: Dabei handelt es sich um Interpunktionszeichen, Diakritika und andere graphische Symbole sowie unterschiedliche Arten der Textmarkierung. Das Ziel der vorliegenden Arbeit besteht darin, die Funktionen von vier ausgewählten graphischen Zeichen (Asteriske, Plus-, Minus- und Gleichheitszeichen) bei Reparaturen in der Kommunikation via IRC-Chat und WhatsApp zu beschreiben. Das Einbeziehen der Daten aus drei Korpora der Mobile Communication Database 1.0 (http://mocoda.spracheinteraktion.de/), der Mobile Communication Database 2.0 (https://db. mocoda2.de/) sowie dem Dortmunder Chat-Korpus (http://www. chatkorpus.tu-dortmund.de/korpora.html) - erlaubt einerseits einen Vergleich der spezifischen Funktionen, die die Zeichen in den

1 Vgl. Pfeiffer (2017) zur Diskussion darüber, dass eine Reihe von lexikalischen Reparaturmarkern einschließlich also als Diskursmarker angesehen werden kann. 
unterschiedlichen Kommunikationsformen ausüben. Andererseits archivieren die Datenbanken Mobile Communication Database 1.0 sowie 2.0 WhatsApp-Daten, die zwischen 2013 und 2018 entstanden sind, sodass auch die Entwicklung des Gebrauchs einzelner Zeichen mikrodiachronisch nachverfolgt werden kann.

\section{Reparaturen: Eine kurze terminologische Einführung}

Eine Reparatursequenz besteht aus drei grundlegenden sequentiellen Komponenten: der Problemquelle (= das Reparandum), der Reparaturinitiierung und der Reparaturdurchführung (vgl. Schegloff et al. 1977; Stukenbrock 2013: 241-242). Die Problemquelle bzw. das Reparandum stellt ein Element innerhalb eines Beitrags eines Interaktionsteilnehmers dar, das als „reparaturbedürftig“ (Stukenbrock 2013: 242) betrachtet wird, d. h. nicht unbedingt als fehlerhaft, sondern als das Aufrechterhalten der Verständigung bedrohend. Daher wird das vorliegende Problem im Laufe der sogenannten Reparaturdurchführung bearbeitet, „der reparierende Ausdruck“ (Pfeiffer 2015: 10) wird dabei als Reparans bezeichnet. Falls eine Reparatur von derselben Person durchgeführt wird, die auch die Problemquelle produziert hat, liegt eine Selbstreparatur vor. Wird das Problem vom Gegenüber bearbeitet, spricht man von einer Fremdreparatur. Der Reparaturdurchführung geht die Reparaturinitiierung voran, d. h. es wird entweder verbal und/oder nonverbal auf das Vorhandensein einer (potentiellen) Problemquelle hingewiesen. Dabei kann eine Reparatur sowohl vom Produzenten als auch vom Rezipienten der Problemquelle initiiert werden (vgl. Pfeiffer 2015: 1-2). Aus unterschiedlichen Zusammensetzungen von Reparaturinitiierungen und -durchführungen ergeben sich vier Reparaturtypen: selbstinitiierte Selbstreparaturen, fremdinitiierte Selbstreparaturen, selbstinitiierte Fremdreparaturen sowie fremdinitiierte Fremdreparaturen (vgl. Schegloff et al. 1977; Stukenbrock 2013: 242-246).

\section{Graphische Ressourcen: theoretischer Überblick}

Bei Reparaturen in der CMC können unterschiedliche Arten der Textmarkierung verwendet werden: Beispielweise kann das Reparandum durchgestrichen (vgl. Piperski/Somin 2014), unterstrichen oder farblich markiert werden (vgl. Vinagre/Muñoz 2011: 76), während das Reparans gelegentlich durch Majuskelschreibung zusätzlich hervorgehoben wird (vgl. Stæhr 2014: 74). Zudem kommen verschiedene nonverbale graphische Zeichen im Rahmen von Repara- 
tursequenzen zum Einsatz. Die häufigsten davon werden im Folgenden kurz dargestellt (für die Beschreibung weiterer Zeichen siehe Mostovaia i. V.).

\subsection{Asterisk}

Das mit Abstand häufigste graphische Zeichen, das in Bezug auf Reparaturen in der Forschungsliteratur erwähnt wird, ist der Asterisk. Seine Funktion als Reparaturmarker ist in mehreren Sprachen (u. a. im Dänischen, Deutschen, Englischen und Russischen) sowie in unterschiedlichen Kommunikationsformen belegt (vgl. Mostovaia i. V.). Die meisten Untersuchungen befassen sich jedoch mit englischsprachigen Daten aus der Chat-Kommunikation. Zu Asterisken als Reparaturmarker im Deutschen liegt nur eine recht überschaubare Anzahl an Arbeiten vor (vgl. Gysin 2015; Schrader 2010).

Eine der ersten Arbeiten, die systematisch auf die Verwendung von Asterisken als Reparaturmarker in der schriftbasierten CMC eingeht, ist die Untersuchung von Collister (2008), die sich mit Reparaturen befasst, die mit einem Asterisk initiiert bzw. markiert werden. Solche Reparaturen werden von ihr anhand von englischsprachigen Chat-Mitschnitten aus dem Online-Spiel World of Warcraft untersucht und als *-repair (star-repair) bezeichnet. Dabei stellt Collister (2008) fest, dass Asteriske i. d. R. für die Korrektur ${ }^{2}$ eines Vertippers oder eines Rechtschreibfehlers benutzt werden.

Laut Collister $(2008$; 2011) kommen Asteriske bei drei von vier Reparaturtypen vor, nämlich bei selbstinitiierten und fremdinitiierten Selbstreparaturen sowie bei fremdinitiierten Fremdreparaturen (nicht aber bei selbstinitiierten Fremdreparaturen), was auch andere Untersuchungen bestätigen (vgl. Gysin 2015; Goloshubina 2016; Stæhr 2014). Am häufigsten finden sich in der Forschungsliteratur Belege für Asteriske, die eine selbstinitiierte Selbstreparatur bzw. -korrektur einleiten wie in (1):

(1) aus Gysin (2015: 224)

$$
\begin{aligned}
& \text { Max }(20: 23: 21): \quad \text { hasch di gesagt } \\
& \operatorname{Max}(20: 23: 27): \quad \mathrm{du}^{*}
\end{aligned}
$$

In Auszug (1) aus einer Chat-Kommunikation über das soziale Netzwerk SchülerVZ vertippt sich Max und gibt in seiner Frage anstatt

2 In der vorliegenden Arbeit werden Fälle, bei denen ein Reparandum bearbeitet wird, das als Verstoß gegen sprachliche Norm angesehen werden kann und daher aus der standardsprachlichen Perspektive als Fehler gelten kann, in Anlehnung an Schegloff et al. (1977) als Korrekturen bezeichnet. Somit werden Korrekturen als Unterkategorie von Reparaturen angesehen. 
von „du“ das Wort „di“ ein, das im Deutschen unter anderem als Abkürzung für Dienstag genutzt wird und deswegen in diesem Kontext ein Verstehensproblem auslösen könnte. Daher leitet Max eine selbstinitiierte Selbstkorrektur ein, indem er das Reparans „du“ postet und dieses mit einem nachgestellten Asterisk versieht. Er signalisiert damit dem Leser, dass ein sprachlicher Ausdruck, nämlich „di“, durch die mit Asterisk gekennzeichnete Form „du“ ersetzt werden soll. Wendet man Reparaturoperationen, die für selbstinitiierte Selbstreparaturen in der gesprochenen Sprache erarbeitet wurden, auf dieses Beispiel an, würde man von einer Substitution sprechen (vgl. Pfeiffer 2015; Schegloff 2013).

Während in (1) eine Substitution vorliegt, handelt es sich in (2) um eine andere Reparaturoperation, nämlich um eine Insertion, d. h. das Einfügen eines Elementes in eine schon bestehende sprachliche Struktur (vgl. Pfeiffer 2015; Schegloff 2013):

(2) aus Goloshubina (2016: 62)

Я уже // испавила // ${ }^{*} \mathrm{p}$

,Hab ich schon // korigiert // ${ }^{*}{ }^{*}$

Beispiel (2) aus einer Instant-Messaging-Kommunikation besteht aus drei Nachrichten, die in der vorliegenden Darstellung durch zwei Schrägstriche voneinander abgetrennt sind und von derselben Person stammen. In der ersten Nachricht wird zuerst über das Abschließen einer Aktivität berichtet, die vermutlich in dem vorherigen Verlauf des Dialogs besprochen wurde. In der darauffolgenden Nachricht wird die Tätigkeit, nämlich das Korrigieren, explizit genannt. Da die eigentlich intendierte Wortform исправила (,korrigiert') durch einen Vertipper zu „испавила“ wird, wird der fehlende Buchstabe „p“ nachgereicht. Der vorangestellte Asterisk hilft dem Rezipienten zu erkennen, dass das ,p“ nicht als ein Teil eines zu früh abgeschickten Beitrags, sondern als eine Selbstkorrektur zu interpretieren ist. Das Wissen über die russische Rechtschreibung erlaubt dem Leser, die Reparaturoperation, für die der Asterisk in dem Fall benutzt wird, als Insertion und nicht als Substitution zu verstehen.

Wie schon erwähnt, werden Asteriske nicht nur bei selbstinitiierten, sondern auch bei fremdinitiierten Selbstreparaturen eingesetzt. In Ausschnitt (3) aus Gysin (2015) kommunizieren zwei befreundete SchülerInnen über die Chat-Funktion eines sozialen Netzwerks. Im Laufe des Dialogs werden unterschiedliche Themen angesprochen, unter anderem das Privatleben von Janosch: 
(3) aus Gysin (2015: 231)

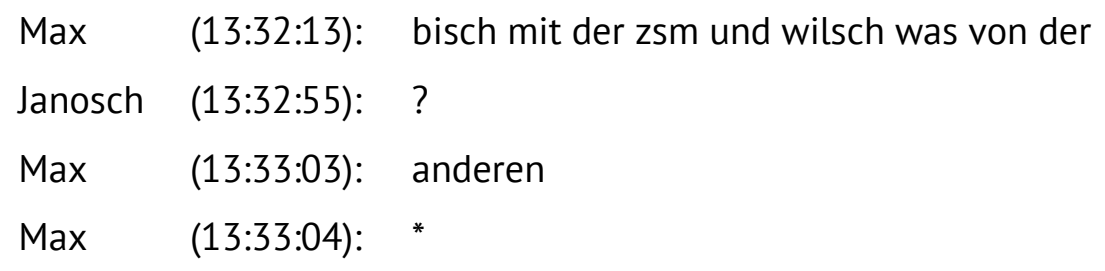

Nach einer langen Diskussion bringt Max die Situation von Janosch auf den Punkt, indem er schreibt, dass Janosch bereits eine Freundin hat, sich jedoch gerade für ein anderes Mädchen interessiert. Dabei löst das zweimal verwendete Demonstrativpronomen der ein Verstehensproblem aus. Janosch signalisiert sein Nicht-Verstehen, indem er in der darauffolgenden Nachricht eine Reparatur durch das Verschicken des alleine stehenden Fragezeichens initiiert, das in dem Fall ähnlich wie ein unspezifischer Reparaturmarker fungiert (vgl. Egbert 2009 sowie das Konzept der 'open' class repair initiators bei Drew 1997). Als Reparaturdurchführung schickt Max zuerst nur das Pronomen „anderen“, das syntaktisch als Fortsetzung seines ursprünglichen Beitrags angesehen werden kann, gefolgt von der Nachricht mit dem alleine stehenden Asterisk, der die durchgeführte Reparatur markiert.

Schließlich können Asteriske auch eine fremdinitiierte Fremdreparatur markieren. Ein solcher Beleg wird in Beispiel (4) aus einem englischsprachigen Chat-Mitschnitt aus World of Warcraft vorgestellt:

(4) aus Collister (2011: 920)

1 1/2 06:16:50.546 [2. Trade] Shak: NEED SOME MORE FOR ZG (=Zul'Gurub) RAID. GOT A HOLE BUNCH!!

2 1/2 06:17:21.375 [2. Trade] Azria: ${ }^{*}$ whole ...

In (4) korrigiert Azria den Tippfehler von Shak, indem sie das richtige Wort „whole“, markiert mit einem vorangestellten Asterisk und drei nachgestellten Punkten, verschickt. Dabei weist Collister (vgl. 2011: 920) darauf hin, dass Shak über längere Zeit mehrere Nachrichten mit dem gleichen Inhalt wie in der Nachricht in der Zeile 1 verschickt und somit ,gespammt' hat, sodass Azria ihre fremdinitiierte Fremdreparatur, die i. d. R. als potentiell gesichtsbedrohende Handlung gilt (vgl. Schegloff et al. 1977), möglicherweise eingesetzt hat, um Shak auf diese Weise implizit zur Ordnung zu rufen.

Die Darstellung von Asterisken bei unterschiedlichen Reparaturtypen liefert wichtige Erkenntnisse zu deren Positionen in Bezug auf 
das Reparans: Asteriske können entweder die vorangestellte (vgl. Beispiele 2 und 4) oder die nachgestellte (vgl. Beispiel 1) Position hinsichtlich des Reparans annehmen. Das Vorkommen des alleinstehenden Asterisks ist m. W. nur bei Gysin (2015) dokumentiert und stellt daher eher einen Sonderfall dar. Ebenfalls relativ selten finden sich in englisch- und dänischsprachigen Daten Fälle, in denen das Reparans von zwei Asterisken umklammert wird (vgl. Christopherson 2013; Darics 2012; Stæhr 2014). Dadurch wird eine Emphase erzeugt, die zusätzliche Aufmerksamkeit auf das Reparans lenkt.

\subsection{Plus-, Minus- und Gleichheitszeichen}

Drei mathematische Zeichen - Plus-, Minus- und Gleichheitszeichen - können ebenso bei Reparaturen eingesetzt werden.

Scheuplein (vgl. 2012: 52-53) führt zwei Beispiele aus einem deutschsprachigen IRC-Chat an, in denen Minuszeichen sowohl alleinstehend (5a) als auch in Kombination mit Pluszeichen (5b) bei Korrekturen verwendet werden:

(5) aus Scheuplein (2012: 53)

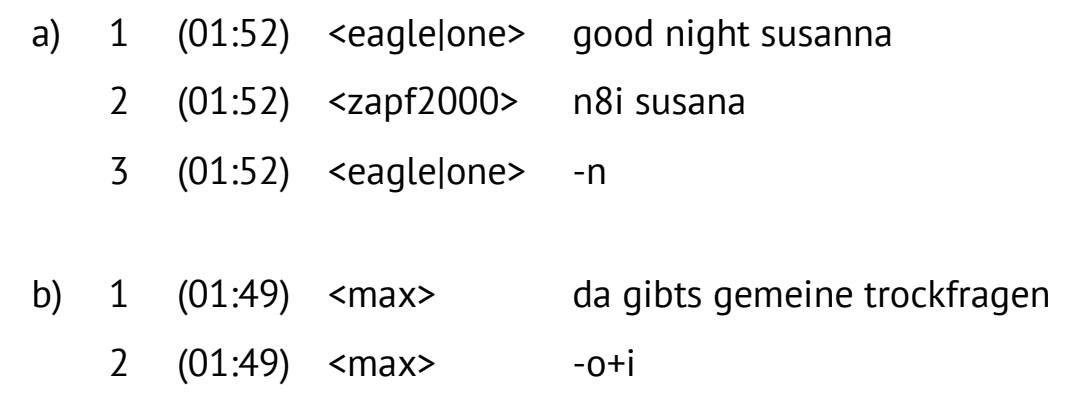

Dabei zeigen Minuszeichen an, dass einzelne Zeichen aus dem vorherigen Beitrag desselben Schreibers getilgt werden sollen, während Pluszeichen die durchgeführte Insertion der darauffolgenden Zeichen kennzeichnen (vgl. Scheuplein 2012: 52-53). Zusammen verwendet erzeugen ein Plus- und ein Minuszeichen eine Substitution wie in (5b).

Gleichheitszeichen finden sich in einem Fall (6) im Kontext von selbstinitiierten Selbstkorrekturen: 
(6) aus Pappert (2017: 189)

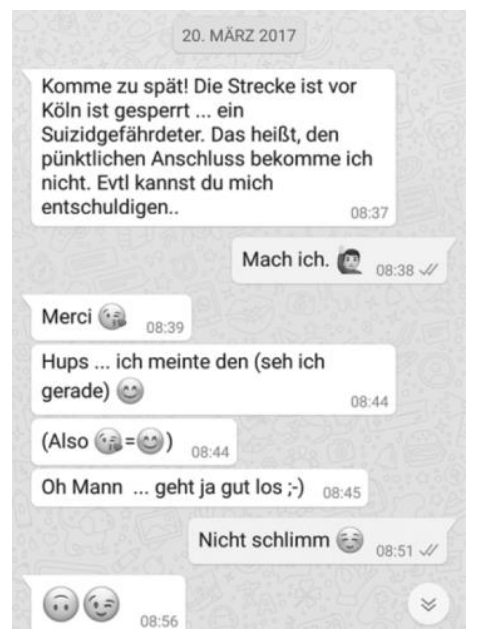

In dem vorliegenden Auszug aus einem WhatsApp-Dialog wird in der dritten Nachricht das falsche Emoji, nämlich ein ,Kuss'-Emoji (3: verschickt. Dieser Fehler wird in der darauffolgenden Nachricht durch die Partikel „Hups“ reflektiert und anschließend in einem für die gesprochene Sprache typischen Format ich meinte $+X$ (vgl. Egbert 2009: 59), in dem $X$ für das Reparans steht, korrigiert (vgl. Pappert 2017: 190). Allerdings reicht diese Reparaturdurchführung der Schreiberin nicht aus, da sie in einem zweiten Anlauf nach einem lexikalischen Reparaturmarker also (vgl. Pfeiffer 2015; 2017) mit Hilfe des Gleichheitszeichens das Reparandum o und das Reparans :noch einmal explizit zueinander in Beziehung setzt (zur Funktion von Klammern vgl. 3.5).

Darüber hinaus berichten Vinagre/Muñoz (vgl. 2011: 76), dass SchreiberInnen in ihren E-Mail-Daten aus einem spanisch-deutschen Sprachtandem bei fremdinitiierten Fremdkorrekturen Reparanda auflisten und jedem davon nach einem Gleichheitszeichen ein Reparans zuordnen.

\subsection{Zirkumflex}

Als nächstes graphisches Zeichen soll der Zirkumflex $\left({ }^{\wedge}\right)$ vorgestellt werden, der in der das Online-Spiel World of Warcraft begleitenden Chat-Kommunikation unter anderem auch bei Reparaturen vorkommt (vgl. Collister 2011; 2012). Dabei soll ein Zirkumflex darauf verweisen, dass das gepostete Reparans zum vorherigen Posting desselben Schreibers gehört wie in (7): 
(7) aus Collister (2012: 14)

1 5/12 00:45:32.242 [Guild] Rufus: they key dodging!

2 5/12 00:45:36.746 [Guild] Rufus: keep^

Im vorliegenden Fall hat sich Rufus bei dem Wort „keep“ vertan und stattdessen „key“ gepostet. Direkt danach initiiert er eine Selbstkorrektur und benutzt einen Zirkumflex als deiktischen Verweis auf das ursprüngliche Posting.

Collister (vgl. 2011: 919) merkt an, dass Zirkumflexe im Vergleich $\mathrm{zu}$ Asterisken selten im Kontext von Reparaturen verwendet werden. In ihrem Chat-Korpus, das sie drei Jahre lang aufgebaut hat und welches über 500.000 Zeilen enthält, treten lediglich 69 von insgesamt 370 Zirkumflexen in Reparatursequenzen auf (vgl. Collister 2012: 18).

\subsection{Pfeil}

Neben Zirkumflexen können auch Pfeile dank ihrer deiktischen Funktion in vergleichbarer Funktion zu Reparaturzwecken eingesetzt werden. Collister (vgl. 2012: 18) beschreibt in ihrem Chat-Korpus zwei Fälle von einem nach links gerichteten Pfeil <--, führt jedoch leider keine Beispiele dafür an, was höchstwahrscheinlich dadurch bedingt ist, dass diese im Vergleich zur Gesamtanzahl von Pfeilen (1141 tokens), die in ihrem Korpus meistens für die Selbstreferenz benutzt werden, ein Randphänomen darstellen.

Ein Beispiel (8) für den Gebrauch eines nach rechts gerichteten Pfeils bei einer Korrektur findet sich bei Kim (2006), die Chat-Mitschnitte von koreanischen EnglischlernerInnen untersucht:

(8) aus Kim (2006: 47-48)

20 CY: I don't know what colander is but I know you are minster haha

$21 \mathrm{CY}$ : sorry minster - > monster

In Zeile 21 korrigiert $C Y$ seinen Vertipper, indem er nach einer Entschuldigung (vgl. apologetic term bei Kitzinger 2013: 240) das Reparandum erneut eingibt und mit dem danach folgenden Pfeil signalisiert, dass dieses als monster verstanden werden soll, es wird also eine Substitution durchgeführt. Diese Strategie könnte man mit dem metasprachlichen Kommentar nicht $X$ (vgl. Pfeiffer 2015: 294) bzw. ich meine nicht $X$, sondern $Y$ (vgl. Egbert 2009) vergleichen. 


\subsection{Klammern}

Lemnitzer/Naumann (2001) befassen sich mit Anakoluthen in einem IRC-Chat im Rahmen eines virtuellen Seminars. Dabei gehen sie an zwei Stellen ihrer Analyse auf die Verwendung von Klammern ein.

Zum einen können Klammern bei einer Retraktion im Sinne einer „Unterbrechung während der Ausführung eines Äußerungsplanes zum Zwecke der Korrektur“ (Lemnitzer/Naumann 2001: 479) eingesetzt werden. Diese Funktion veranschaulichen sie mit Hilfe des folgenden Chatausschnitts (9):

(9) aus Lemnitzer/Naumann (2001: 479)

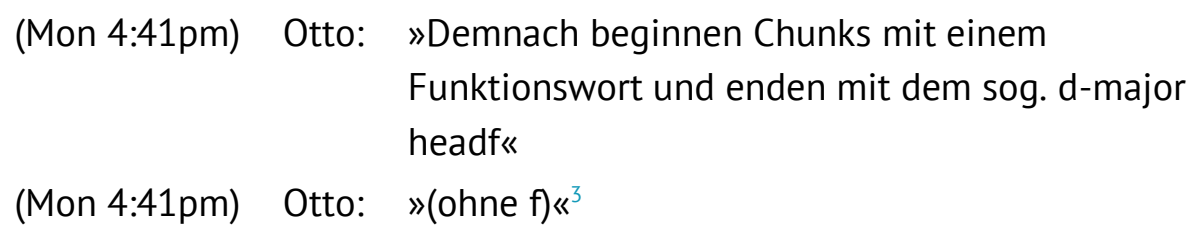

Die in Klammern stehende Präposition „ohne“ sowie der Buchstabe „f“ geben dem Rezipienten den Hinweis darauf, dass das $f$ aus einem Wort aus dem davorstehenden Beitrag getilgt werden soll. Dafür kommt nur das Wort „headf“ in Frage, das als head verstanden werden soll.

Die Verwendung von Klammern bei der Retraktion in (9) kann darüber hinaus auf eine von zwei Funktionen von Klammern zurückgeführt werden, die Lemnitzer/Naumann (vgl. 2001: 481-483) im Verlauf ihrer Untersuchung herausarbeiten. Dabei handelt es sich zum einen um die Möglichkeit mit Klammern, eine eingeschobene Äußerung, die eine andere illokutive Funktion als der Rest des Beitrags erfüllt, in diesen zu integrieren und gleichzeitig davon abzutrennen (vgl. Lemnitzer/Naumann 2001: 482).

Des Weiteren dienen Klammern in der Chat-Kommunikation dazu, „ein zur geplanten Proposition gehörendes“, aber syntaktisch nicht (mehr) passendes Element nachzureichen (Lemnitzer/Naumann 2001: 482). Ein solcher Fall findet sich in Ausschnitt (10), in dem die Adverbphrase „schon vorher“, die eigentlich ins Mittelfeld gehört, nachgeliefert und daher eingeklammert wird:

3 Die Anführungszeichen sind dem Original entnommen. Diese scheinen allerdings zu den Notationskonventionen zu gehören, da jeder Chat-Beitrag in den bei Lemnitzer/Naumann (2001) präsentierten Daten in doppelte Anführungszeichen eingeschlossen ist. 
(10) aus Lemnitzer/Naumann (2001: 482)

(Mon 3:37pm) Ludwig: "Deshalb habe ich mich mit Thomas zusammengesetzt (schon vorher)《

Die Frage, warum Ludwig in dem Fall keine Revision (zu Revisionen vgl. Beißwenger 2010) vorgenommen und die eingeklammerte Phrase nicht direkt in das Mittelfeld integriert hat, bleibt offen. Für das Erscheinen von „schon vorher“ könnte man zum einen mit den Anforderungen an die schnelle Beitragsproduktion argumentieren, die durch die zweifache Veränderung der Position der Schreibmarke verlangsamt wird. Zum anderen handelt es sich hier eigentlich um einen Nachtrag, der als eine nicht-kanonische Nachfeldbesetzung interpretiert werden könnte (vgl. Imo 2015a). Der fehlende Kontext lässt allerdings die mögliche Funktion dieser Nachfeldbesetzung nicht eindeutig bestimmen.

\subsection{Fragezeichen}

Alleine stehende Fragezeichen als Fremdinitiierungen können das allgemeine Nicht-Verstehen eines der vorangehenden Beiträge thematisieren, wie in Beispiel (3), das bereits in der vorliegenden Untersuchung analysiert wurde (weitere Beispiele finden sich in Cherny 1999: 117; Stæhr 2014: 85). In dieser Funktion ähneln Fragezeichen gesprochensprachlichen unspezifischen Reparaturinitiierungen (vgl. Egbert 2009) bzw. ,open' class repair initiators (vgl. Drew 1997), deren Funktion darin besteht, ein Problem des Hörens oder des Verstehens anzuzeigen, jedoch ohne dass die Problemquelle genauer lokalisiert oder die Art des Problems näher bestimmt wird (vgl. Egbert 2009: 99; Drew 1997). Da aber in der CMC - abgesehen von Sprachnachrichten und Anrufen - das Rezipieren des Beitrags grundsätzlich durch das Medium der Schrift gesichert ist, beschränkt sich die Verwendung von Fragezeichen auf das Anzeigen von Verstehensproblemen. Des Weiteren können potentiell die sogenannten Erwartungsprobleme mit Fragezeichen signalisiert werden, d. h. diejenigen Probleme, bei denen das geäußerte Reparandum in Widerspruch zu den Erwartungen bzw. dem Wissensstand der RezipientInnen steht (vgl. Couper-Kuhlen/Selting 2018: 188195). 


\section{Daten}

Für die empirische Analyse der graphischen Ressourcen bei Reparaturen in der informellen Schriftlichkeit wurden Daten aus drei linguistischen Korpora herangezogen: der Mobile Communication Database 1.0 (http://mocoda.spracheinteraktion.de/), der Mobile Communication Database 2.0 (https://db.mocoda2.de/) und dem Dortmunder Chat-Korpus (http://www.chatkorpus.tu-dortmund.de/).

Die Mobile Communication Database 1.0 (MoCoDa 1.0) enthält deutsch- und russischsprachige (dyadische) Dialoge via SMS sowie per WhatsApp und anderen internetbasierten Messengerdiensten mitsamt den Metadaten der SchreiberInnen. Dabei sind in der MoCoDa 1.0 Nachrichten archiviert, die zwischen 2013 und 2017 ausgetauscht wurden. Das deutsche Subkorpus bestand zum Zeitpunkt der Untersuchung aus 2.204 Dialogen bzw. 19.210 Nachrichten. Für die Analyse wurden nur deutsche Dialoge herangezogen, die über den Messengerdienst WhatsA $P P$ verschickt wurden. Der Umfang des dadurch zusammengestellten Subkorpus beläuft sich auf 805 WhatsApp-Dialoge (9289 Nachrichten). Die Mobile Communication Database 2.0 (MoCoDa 2.0) - Nachfolgeversion von MoCoDa 1.0 - beinhaltet nun sowohl dyadische Interaktionen als auch GruppenChats via WhatsApp. Zur Zeit der Recherche umfasste die MoCoDa 2.0171 WhatsApp-Chats bzw. 18106 Nachrichten, die im Jahre 2018 verschickt wurden.

Da der größte Teil der Kurznachrichtendialoge aus der MoCoDa 1.0 und 2.0 von Studierenden stammt und informellen Charakters ist, wurden zur besseren Vergleichbarkeit aus dem Dortmunder Chat-Korpus Ausschnitte aus der Plauder-Chat-Umgebung SpaceChat auf www.unicum.de ausgewählt, die ebenso von Studierenden sowie von SchülerInnen der gymnasialen Oberstufe stammen. Das Subkorpus enthält 15481 Chat-Beiträge (http://www.chatkorpus.tudortmund.de/files/bestandsliste-gesamtkorpus.pdf).

\section{Graphische Ressourcen im Korpus}

Die in Abschnitt 4 beschriebenen Korpora wurden manuell durchsucht. Die Suchergebnisse sind in der Tabelle unten zusammengefasst, aus der hervorgeht, dass sich sowohl Gemeinsamkeiten als auch Unterschiede in der Verwendung und Verteilung von graphischen Zeichen in der Kommunikation via IRC-Chat und WhatsApp im Deutschen zeigen. 


\begin{tabular}{|c|c|c|c|}
\hline Zeichen & $\begin{array}{l}\text { Dortmunder } \\
\text { Chatkorpus } \\
\text { (Unicum) }\end{array}$ & $\begin{array}{l}\text { Mobile Com- } \\
\text { munication } \\
\text { Database } 1.0\end{array}$ & $\begin{array}{l}\text { Mobile Com- } \\
\text { munication } \\
\text { Database } 2.0\end{array}$ \\
\hline Asteriske & 1 & 14 & 38 \\
\hline Klammern & 8 & 3 & 2 \\
\hline Fragezeichen & 5 & 6 & 8 \\
\hline Pluszeichen & $12(+2)$ & - & - \\
\hline Minuszeichen & $12(+2)$ & - & 1 \\
\hline Gleichheitszeichen & 10 & 1 & 1 \\
\hline Bindestriche & - & 1 & - \\
\hline \multirow[t]{2}{*}{ Anführungszeichen } & 19 & 5 & 6 \\
\hline & $\begin{array}{r}69 \\
\text { (in 15481 } \\
\text { Beiträgen) }\end{array}$ & $\begin{array}{r}30 \\
\text { (in } 9289 \\
\text { Nachrichten) }\end{array}$ & $\begin{array}{r}56 \\
\text { (in } 18106 \\
\text { Nachrichten) }\end{array}$ \\
\hline
\end{tabular}

Tabelle: Anzahl graphischer Ressourcen bei Reparaturen in den Untersuchungskorpora

Die Suche ergab, dass in keinem der Untersuchungskorpora Zirkumflexe oder Pfeile zu Reparaturzwecken verwendet werden. Hier muss jedoch angemerkt werden, dass Pfeile dennoch in den im Rahmen dieser Arbeit nicht untersuchten Subkorpora des Dortmunder Chat-Korpus eine reparative Funktion erfüllen (vgl. Mostovaia i. V.). Auch Reparaturen mit Textmarkierungen (Unterstreichen, Durchstreichen, Farbmarkierung etc.) finden sich nicht, während die Majuskelschreibung in zwei Fällen bei den WhatsApp-Daten zum Einsatz kommt (zur Analyse dieser Fälle s. Mostovaia i. V.). Die Tatsache, dass auf die Textmarkierungen nicht zurückgegriffen wird, könnte einerseits damit zu tun haben, dass diese Funktionen z. T. technisch nicht oder nur eingeschränkt umsetzbar sind, wie z. B. die Farbmarkierung $^{4}$ in der WhatsApp-Kommunikation. Andererseits stellt sich eine Textmarkierung v. a. bei der Eingabe einer WhatsApp-Nachricht aufwendig dar: Um einen entsprechenden Textabschnitt beispielsweise durchzustreichen, muss dieser zwischen zwei

4 Vgl. dazu die Informationen auf der WhatsApp-Seite: https://www.whatsappsim.de/ratgeber/whatsapp-faq/whatsapp-schriftfarbe-und-schriftart-aendern. 
Tilden gesetzt werden. ${ }^{5}$ Außerdem könnte das Ausbleiben von Textmarkierungen an der technischen Beschaffenheit der herangezogenen Datenbanken liegen, die diese Textmarkierungen schlichtweg nicht abbilden können.

Neben den bereits im theoretischen Teil dieser Arbeit beschriebenen graphischen Ressourcen wurden in den untersuchten Daten Belege gefunden, in denen ein weiteres Interpunktionszeichen, nämlich Anführungszeichen, bei verbalen Initiierungen eingesetzt wurde, um beispielsweise das wiederaufgenommene Reparandum („Was heißt denn „hier“?“” (\#XyT2E, MoCoDa 2.0)) zu kennzeichnen oder ein Verstehensversuch (candidate understanding im Sinne von Schegloff 1997) zu präsentieren („Moritz meinte wohl "gekotzt hab"““ (\#fhLyA, MoCoDa 2.0)). Im ersten Fall treten Anführungszeichen in ihrer klassischen Verwendungsweise auf und zeigen einen übernommenen Abschnitt ( $\approx$ ein Zitat) an, während im zweiten Fall Anführungszeichen zusätzlich dazu beitragen, sich vom Inhalt der angebotenen Verstehensinterpretation zu distanzieren. Darüber hinaus wurde in einem WhatsApp-Dialog aus der MoCoDa 1.0 ein Bindestrich entdeckt, der einen Wortabbruch bei einer Reparatur markiert.

In der vorliegenden Arbeit sollen nun die Suchergebnisse für folgende graphische Ressourcen ausgewertet werden: Asteriske, Plus-, Minus- sowie Gleichheitszeichen. Die Verwendungsweisen von Klammern sowie Fragezeichen in den Untersuchungskorpora ähneln weitgehend den in der Forschungsliteratur (vgl. Abschnitte 3.5 und 3.6) beschriebenen Funktionen und werden ausführlich in Mostovaia (i. V.) diskutiert.

\subsection{Asterisk}

Asteriske kommen zwar bei Reparaturen in allen drei Korpora vor, weisen allerdings dabei eine ungleichmäßige Verteilung auf. Während Asteriske im untersuchten IRC-Chat äußerst selten sind (ein einziges Vorkommen eines alleinstehenden Asterisks), machen sie in der WhatsApp-Kommunikation hingegen den größten Teil der für Reparaturzwecke verwendeten graphischen Ressourcen aus.

In den WhatsApp-Daten aus der MoCoDa 1.0 und 2.0 werden 52 Reparaturen mit einem Asterisk markiert, eine davon stammt jedoch aus einem Dialog, der in englischer Sprache verfasst wurde, sodass diese in der Analyse nicht mitberücksichtigt wird. Die somit analysierten 51 Asteriske verteilen sich auf drei Reparaturtypen: Sie kommen bei selbstinitiierten und fremdinitiierten Selbstreparaturen sowie bei fremdinitiierten Fremdreparaturen vor (vgl. 3.1). Dabei zeigt

5 S. Fußnote 4. 
sich, dass Asteriske weitaus häufiger bei selbstinitiierten Selbstreparaturen gebraucht werden: Diese Funktion erfüllen die Mehrzahl der Asteriske aus den WhatsApp-Daten (46 tokens, $87 \%$ aller Asteriske als Reparaturmarker) sowie der einzige Asterisk aus dem Dortmunder Chat-Korpus. Im Vergleich dazu finden sich Asteriske bei fremdinitiierten Selbst- und Fremdreparaturen selten: In den untersuchten WhatsApp-Daten liegen lediglich jeweils 2 und 3 Fälle vor. In drei weiteren Abschnitten sollen einzelne Aspekte von Reparaturen mit Asterisken (wie z. B. das vorliegende Reparandum, die Reparaturoperation, die Position der Asteriske in Bezug auf das Reparans etc.) näher beschrieben werden.

\subsubsection{Asteriske bei selbstinitiierten Selbstreparaturen}

\subsubsection{MoCoDa 1.0 und 2.0}

Die Mehrheit der im Untersuchungskorpus vorgefundenen Asteriske bilden diejenigen, die eine selbstinitiierte Selbstreparatur einleiten. Dabei handelt es sich vor allem um solche Reparatursequenzen, bei denen das Reparandum (oft als Produkt eines Vertippers oder eines nicht erwünschten Eingriffs des Autokorrekturprogramms) einen Fehler darstellt, sodass man streng genommen von selbstinitiierten Selbstkorrekturen ausgehen muss (vgl. Fußnote 2).

Der Asterisk scheint ein polyfunktionaler Reparaturmarker zu sein, mit dessen Hilfe verschiedene Problemquellen bearbeitet werden können: von einzelnen Wörtern sowie Morphemen über Interpunktionszeichen und Zahlen bis zu Emoticons und Emojis. Wenn man die an die sprachlichen Ebenen angelehnte Typologie der Reparanda in der gesprochenen Sprache (vgl. Pfeiffer 2015: 54) an die Analysedaten anpasst, kann man von folgenden Reparaturtypen ausgehen: (ortho)graphische, morphosyntaktische, lexiko-semantische und pragmatische. Darüber hinaus liegen im Untersuchungskorpus einige Fehler vor, die man nicht als sprachliche, sondern eher als logische bzw. inhaltliche Fehler bezeichnen würde. Solche Reparatur-Kategorisierungen sind allerdings nur als Prototypen zu sehen. Genau wie in der gesprochenen Sprache (vgl. Pfeiffer 2015) lassen sich auch in der geschriebenen Sprache die Fälle meist nicht klar einer Gruppe zuordnen, sondern mehreren. Kontrovers sind zum Beispiel Fälle, bei denen denn durch den und umgekehrt ausgetauscht werden. Zur Veranschaulichung werden im Folgenden Belege für je eine morphosyntaktische sowie eine pragmatische Korrektur präsentiert.

Morphosyntaktische Korrekturen im Analysekorpus betreffen u. a. Fälle, bei denen entweder eine fehlerhafte Kongruenz vorliegt oder ein obligatorisches syntaktisches Element in einer Nachricht nicht realisiert wird, wie in (11): 
(11) aus der Mobile Communication Database 2.0: Auszug aus dem WhatsApp-Dialog \#tmMCy

$$
\begin{aligned}
& \text { Wo wart inr was trinken? } \\
& \text { Leyla (\#331 - 13.03.2018 - 21:27) }
\end{aligned}
$$

Ähm im Celona hier in Wandsbeck... war ganz cool dort

Florian (\#332 - 13.03.2018 - 21:27)

Da kann übrigens super Brunchen am Wochenende d)

Florian (\#333 - 13.03.2018 - 21:28)

${ }^{*}$ man

Florian (\#334 - 13.03.2018 - 21:28)

Jaa da war ich auch schonmal

Leyla (\#335 - 13.03.2018 - 21:28)

Leyla und Florian daten. Im vorherigen Verlauf des Dialogs (\#319, nicht abgebildet) teilt Florian mit, dass er mit ein paar Freunden in einer Bar sitzt. In \#331 fragt Leyla danach, in welcher Bar Florian gewesen ist. Nach seiner Antwort in \#332 liefert Florian in \#333 noch eine zusätzliche Information über diese Bar, nämlich, dass da am Wochenende guter Brunch serviert wird. In \#333 fehlt allerdings das Subjekt, das in \#334 nachgereicht wird. Somit liegt hier eine Insertion vor, bei der das Reparans mit einem vorangestellten Asterisk markiert wird.

Eine pragmatische Korrektur liegt in (12) vor. Alexander veranstaltet bei sich zu Hause eine Party. Sandra ist eingeladen und fragt den Gastgeber nach der Haltestelle, neben der er wohnt.

(12) aus der Mobile Communication Database 2.0: Auszug aus dem WhatsApp-Gruppenchat \#7YVuf

Wie heisst deine haltestelle?

Sandra (Nachricht \#58 - 20.08.2016 - 16:23) 


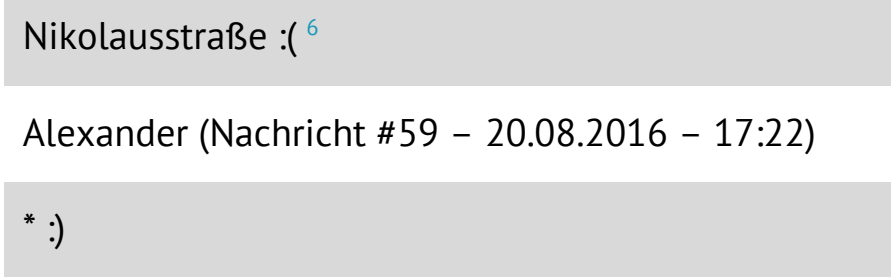

Alexander (Nachricht \#60 - 20.08.2016 - 17:22)

Thx :*

Sandra (Nachricht \#61 - 20.08.2016 - 17:23)

Alexander liefert die von Sandra angefragte Information (den Haltestellenamen) in \#59 versehentlich mit dem Emoticon ,:(“, das in dem Fall beispielsweise seine Unzufriedenheit mit seinem Wohnort bzw. mit der Lage der Haltestelle zum Ausdruck bringen könnte. Das Emoticon wird von Alexander umgehend nach dem Verfassen der Nachricht korrigiert. Dafür verschickt er das Emoticon „:)“ mit einem vorangestellten Asterisk.

In einem Gruppenchat (13) handeln Schreiber die Zeit für ein gemeinsames Treffen aus. Während Janus in \#1452 mitteilt, dass er zeitlich flexibel sei, äußert Bernd zwar seinen Wunsch mitzukommen, gibt aber zu bedenken, dass er erst nach seinem Training Zeit hat (\#1453):

(13) aus der Mobile Communication Database 2.0: Auszug aus dem WhatsApp-Gruppenchat \#fhLyA

Bin da flexibel, können irgendwie gegen 7 oder so los, aber wenn mehrere erst später wollen geht auch 8, halb 9

Janus (Nachricht \#1452 - 20.04.2018 - 13:32)

Würde mitkommen, trainiere aber erst bei der dritten mit

Bernd (Nachricht \#1453 - 20.04.2018 - 13:36)

6 Der im Original stehende anonymisierte Ausdruck „Straße“ wurde hier zur besseren Verständlichkeit durch einen fingierten Straßennamen ersetzt. 


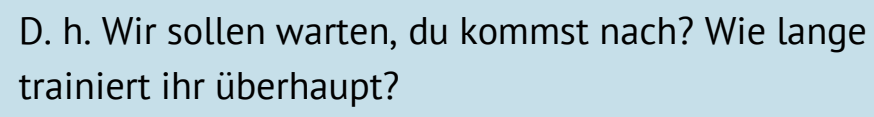

Janus (Nachricht \#1454 - 20.04.2018 - 13:48)

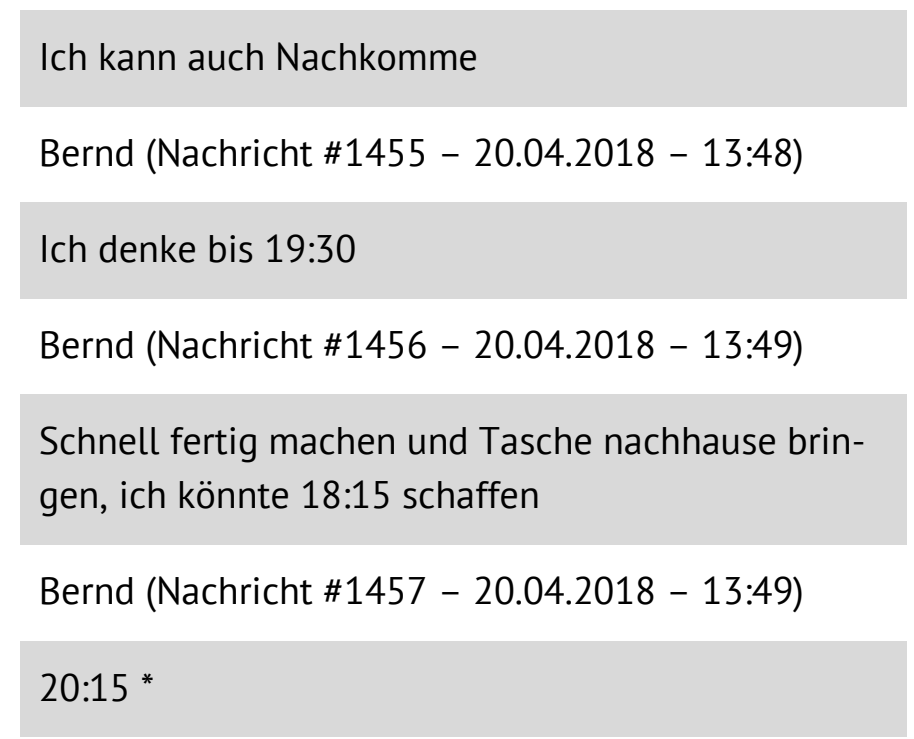

Bernd (Nachricht \#1458 - 20.04.2018 - 13:49)

In \#1454 könnte man die erste Frage von Janus als eine Fremdinitiierung zu Bernds Nachricht \#1453 deuten, die in Form eines candidate understanding, eingeführt mit das heißt, Bernds Nachricht auf der Handlungsebene hinterfragt: Bedeutet deine Nachricht, dass du nur etwas später kommst oder dass wir wegen dir die Zeit verschieben müssen? Außerdem stellt Janus in der gleichen Nachricht eine Frage danach, wie lange Bernds Training dauere. In \#1455 bestätigt Bernd, dass er nachkommen kann, dann teilt er in \#1456 mit, dass sein Training gegen 19:30 zu Ende sein müsse. Anschließend erläutert er, dass er nach dem Training lediglich seine Tasche nach Hause bringen müsse und 18:15 zu den anderen dazustoßen würde. Diese Zeitangabe (18:15) widerspricht jedoch der bereits in \#1456 gelieferten Information zum Trainingsende (19:30), sodass ein logischer Fehler vorliegt. Dieser wird in \#1458 von Bernd im Laufe einer selbstinitiierten Selbstkorrektur wiedergutgemacht, indem er die zweite Zeitangabe „18:15“ durch die korrekte Angabe „20:15“ ersetzt und das Reparans mit einem vorangestellten Asterisk markiert.

Beispiele (11)-(13) machen deutlich, dass bei selbstinitiierten Selbstkorrekturen Asteriske sowohl bei Substitutionen als auch bei Insertionen verwendet werden. Dabei kommen Asteriske bei Substitutionen wesentlich häufiger als bei Insertionen vor (jeweils 38 und 8 Fälle). Bei Substitutionen können mit Asterisken gekenn- 
zeichnete Reparantia ganze Wörter, Morpheme, einzelne Buchstaben innerhalb eines Wortes, Interpunktionszeichen, Emojis und Emoticons ersetzen. Bei Insertionen werden mit einer Ausnahme einzelne Wörter in die bereits bestehende syntaktische Struktur einer Nachricht eingefügt. Lediglich in einem Chat-Verlauf (\#KAU9B, MoCoDa 2.0) fungiert ein einzelner Buchstabe als Reparans. Auffällig ist, dass alle acht Fälle mit Insertionen aus der MoCoDa 2.0 stammen und WhatsApp-Verläufen entnommen sind, die 2018 entstanden sind. Die Frage, ob frühere WhatsApp-Daten aus dem Untersuchungskorpus lediglich zufällig keine mit Asterisken gekennzeichneten Insertionen nachweisen oder ob die Verwendung von Asterisken in solchen Fällen sich mit der Zeit entwickelt hat, muss offenbleiben.

Aus der zeitlichen Perspektive erfolgt die Reparaturinitiierung und -durchführung in den untersuchten Daten sehr schnell: Bei 32 Fällen innerhalb der gleichen und bei 11 Fällen innerhalb der nächsten Minute nach dem Abschicken der Nachricht, die die Problemquelle enthält. Dadurch wird die Nachricht mit dem Reparans sequentiell nah am Reparandum platziert: 41 von 46 selbstinitiierten Selbstreparaturen werden direkt in der darauffolgenden Nachricht initiiert und durchgeführt. Die Tatsache, dass dafür die Tastatur umgeschaltet werden muss (die Zeichentastatur muss aktiviert werden) scheint dabei so wenig Zeit in Anspruch zu nehmen, dass Asteriske trotzdem nah am jeweiligen Reparans platziert werden können.

In den bereits präsentierten Beispielen mit Asterisken als Reparaturmarker wird bei der Reparaturdurchführung lediglich das jeweilige Reparans geliefert. Dabei wird beispielsweise bei Substitutionen das zu ersetzende Reparandum nicht explizit genannt, sodass dieses von den RezipientInnen der Nachricht selbst identifiziert werden muss, indem z. B. morphosyntaktische Eigenschaften des Reparandums sowie seine partielle graphische Ähnlichkeit mit dem Reparans als Hilfestellung benutzt werden können. Bei Insertionen, bei denen eine obligatorische Konstituente wie z. B. das Subjekt (Beispiel 11) eingefügt werden muss, orientiert man sich an der erwartbaren syntaktischen Struktur einer Nachricht. In 6 von 46 Fällen wird allerdings bei der Reparaturdurchführung ein Teil der Nachricht mit der Problemquelle wiederholt und somit auf den präpositionierten oder postpositionierten syntaktischen Anker zurückgegriffen, um den RezipientInnen die Aufgabe zu erleichtern, das Reparans in die ursprüngliche syntaktische Struktur der Nachricht richtig zu integrieren. Es liegen drei Fälle mit einem postpositionierten und ein Fall mit einem präpositionierten syntaktischen Anker vor. Bei zwei weiteren Fällen - darunter auch (14) - tritt eine Kombination aus einem post- und einem präpositionierten Anker auf. Bei 
der Unterbreitung eines Vorschlags, ein Bier trinken zu gehen, verwendet Bernd in der Präpositionalphrase „an ein Bier“ (\#518) den unbestimmten Artikel unkorrekterweise im Akkusativ. Obwohl Markus in \#519 auf seinen Vorschlag angemessen reagiert und somit implizit anzeigt, dass der vorliegende grammatische Fehler das Verständnis der Nachricht nicht beeinträchtigt, wird dieser in \#520 trotzdem von Bernd behoben:

(14) aus der Mobile Communication Database 2.0: Auszug aus dem WhatsApp-Gruppenchat \#Qy1Pp

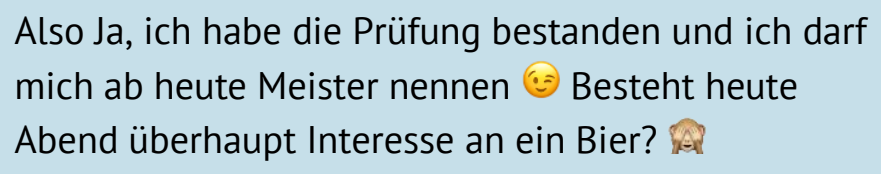

Bernd (Nachricht \#520 - 01.02.2018 - 16:34)

Im Laufe der Reparaturdurchführung verschickt Bernd allerdings nicht das alleinstehende Reparans, sondern retrahiert zum Kopf der Präpositionalphrase und gibt die ganze Phrase erneut ein, sodass das Reparans „einem“ mit dem präpositionierten „an“ und dem postpositionierten „Bier“, die gemeinsam den syntaktischen Anker bilden, eingeklammert wird.

Aufgrund der geringen Anzahl von Beispielen lässt sich zurzeit noch kein Zusammenhang zwischen dem Vorkommen eines syntaktischen Ankers und der durchgeführten Reparaturoperation (2 Fälle bei Insertionen und 4 bei Substitutionen) oder der Position bzw. dem Typ des Reparandums in der Ausgangsnachricht erkennen. Der Frage, wodurch der Einsatz eines syntaktischen Ankers bedingt ist, muss daher in zukünftigen Untersuchungen nachgegangen werden.

In Bezug auf die Position des Asterisks lassen sich diejenigen Positionen, die bereits für englisch- sowie russischsprachige Daten beschrieben wurden (vgl. 3.1), auch in den deutschen WhatsApp-Verläufen wiederfinden. In der Mehrheit der Belege wird das Reparans mit einem vorangestellten Asterisk markiert (32 von 46 Fällen), während bei 13 selbstinitiierten Selbstkorrekturen der Asterisk eine 
nachgestellte Position in Bezug auf das Reparans annimmt. Darüber hinaus findet sich ein Fall, bei dem das Reparans von beiden Seiten mit Asterisken „eingeklammert“ wird (\#4I1Cp, MoCoDa 2.0).

Unabhängig von seiner Position macht der Asterisk in der MoCoDa 1.0 und 2.0 ein Reparans kenntlich, das entweder durch Substitution oder durch Insertion in die syntaktische Struktur einer der vorherigen Nachrichten integriert werden soll. Dabei scheint die Position der Asteriske in Bezug auf das Reparans keine distinktive pragmatische Funktion auszuüben, sondern eher einen idiosynkratischen Charakter zu haben. Das lässt sich beispielsweise bei der Analyse von zwei Gruppenchats (\#fhLyA und \#Qy1Pp) erkennen, die von den gleichen SchreiberInnen stammen und in denen sechs von 14 bzw. 15 ${ }^{7}$ SchreiberInnen mehr als eine Reparatur mit einem Asterisk entweder in ausschließlich vorangestellter oder in ausschließlich nachgestellter Position markieren. Diese Hypothese der idiosynkratischen Verwendung von * müsste jedoch an einer größeren Anzahl von Beispielen überprüft werden.

\subsubsection{Dortmunder Chat-Korpus}

Im Chat-Subkorpus Unicum lässt sich nur ein einziges Beispiel finden, in dem der alleinstehende Asterisk analog zu (11)-(13) als Reparaturmarker benutzt wird. In Auszug (15) stellt zora eine Frage nach den Kosten eines Umzugs mit einem Umzugsunternehmen. Diese wird von Shadok in Zeile 208 ironisch beantwortet:

(15) aus dem Dortmunder Chat-Korpus: Auszug aus Dokument 2221006_ unicum_21-02-2003_(1).xml

$\begin{array}{lll}203 & \text { zora } & \text { weiß jetzt jemand was umzugsunternehmen so kosten? } \\ 204 & \text { quaki } & \text { danke shadok } \\ 205 & \text { Shadok } & \text { bitte schön. } \\ 206 & \text { Shadok } & \text { heya Lanto ;0) } \\ 207 & \text { Lantonie } & \text {;0) } \\ 208 & \text { Shadok } & \text { geld zorsa } \\ 209 & \text { Shadok } & \text { *zora } \\ 210 & \text { Pharao } & \text { teuer.geht nich selber machen mit mietwagen? } \\ 211 & \text { Shadok } & \text { meistens euro } \\ 212 & \text { Shadok } & \text {;0) } \\ 213 & \text { zora } & \text { verdammt, das hab ich befürchtet shaddy :P }\end{array}$

7 In \#fhLyA wurde ein weiterer Schreiber dem Gruppenchat hinzugefügt. 
Bei zoras Nickname unterläuft Schadok ein Tippfehler, den er korrigiert, indem er den richtigen Nickname mit einem vorangestellten Asterisk im nächsten Beitrag verschickt. ${ }^{8}$

Das äußerst seltene Vorkommen von Asterisken als Reparaturmarker im Unicum-Korpus im Gegensatz zu den analysierten WhatsApp-Daten kann auf unterschiedliche Ursachen zurückgeführt werden. Zum einen kann es an der in der Chat-Kommunikation via IRC-Chat herrschenden Konvention liegen, Inflektive sowie Inflektivkonstruktionen mit einem oder zwei Asterisken zu markieren. Diese fängt laut Henn-Memmesheimer/Eggers (2010) erst 2004 an, sich peu à peu aufzulösen. Dabei könnte eine parallel existierende Konvention, das Reparans bei einer Reparatur bzw. Korrektur mit einem Asterisk zu kennzeichnen, zur Verwirrung führen. Gleichzeitig lassen sich allerdings in der Community aus dem Unicum-Chat andere Strategien nachweisen, mit deren Hilfe die gleichen Reparaturoperationen, die potentiell mit Asterisken angezeigt werden können, durchgeführt werden (s. 5.2 und 5.3). Leider liegen keine Daten zum Ursprung sowie zum Aufkommen der Konvention vor, Asteriske als Reparaturmarker einzusetzen, sodass nicht ausgeschlossen werden kann, dass diese Verwendungsweise zur Zeit der Datenerhebung im deutschsprachigen Raum noch nicht existierte bzw. nicht weit verbreitet war. Außerdem fällt auf, dass Asteriske als Reparaturmarker oft in solchen Chat-Kommunikationen, die ein Online-Spiel begleiten, beschrieben werden (vgl. Collister 2008; 2011; Schrader 2010). Man könnte daher annehmen, dass sich die Funktion der Asteriske, eine Reparatur zu markieren, in dieser Chat-Gattung herausgebildet hat und sich allmählich in anderen Gattungen und Kommunikationsformen ausgebreitet hat.

\subsubsection{Asteriske bei fremdinitiierten Fremdreparaturen}

Zur Markierung von fremdinitiierten Fremdreparaturen fanden sich in den analysierten WhatsApp-Daten drei Belege. Dabei handelt es sich um zwei Korrekturen und eine Elaborierung (vgl. Pfeiffer 2015), die in eine Scherzaktivität miteinbezogen ist (vgl. \#Qy1Pp, MoCoDa 2.0). In Bezug auf die durchgeführte Reparaturoperation stellen die beiden Korrekturen Substitutionen dar, in deren Rahmen orthographische Reparanda bearbeitet werden (vgl. 16). Bei der Elaborierung handelt es sich um eine Insertion einer nicht obligatorischen syntaktischen Konstituente.

8 Die Zeitangaben für Chat-Verläufe aus dem Dortmunder Chat-Korpus stehen leider nicht zur Verfügung, sodass hier keine Aussagen in Bezug auf die Zeitlichkeit der vorliegenden Reparaturinitiierung bzw. -durchführung getroffen werden können. 
In (16) planen die Schreiberinnen einen gemeinsamen Abend. Im vorherigen Verlauf des Dialogs erkundigt sich Jana, welchen Käse sie für die Pizza kaufen soll: Streukäse oder Mozzarella. In \#126 stellt sie klar, dass sie bei Streukäse an Gouda gedacht hat, schreibt allerdings den Käsenamen falsch, nämlich „Gauda“:

(16) aus der Mobile Communication Database 2.0: Auszug aus dem WhatsApp-Gruppenchat \#9GGAT

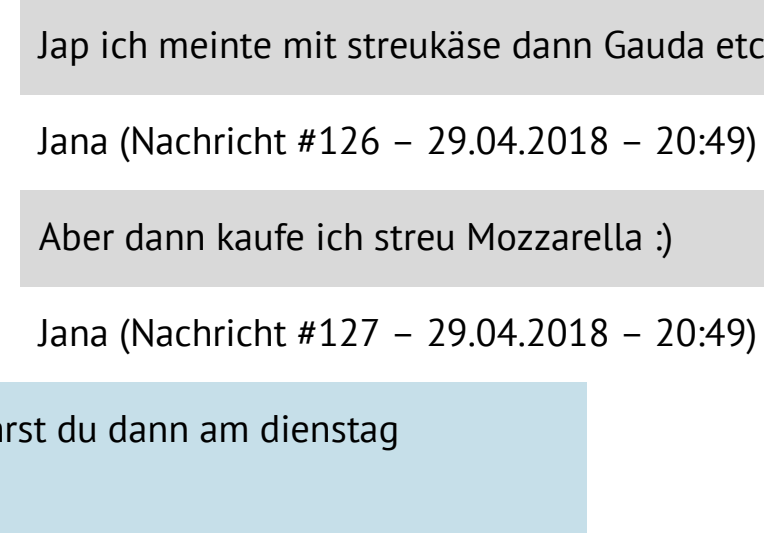

Andrea (Nachricht \#128 - 29.04.2018 - 20:51)

Gauda :D

Clara (Nachricht \#129 - 29.04.2018 - 20:55)

Ja streugauda

Jana (Nachricht \#130 - 29.04.2018 - 21:00)

\section{*Gouda (6)}

Lili (Nachricht \#131 - 29.04.2018 - 21:30)

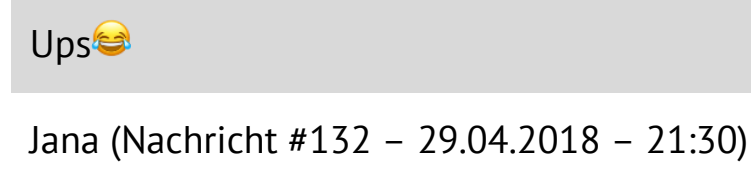

In \#129 versucht Clara, Jana auf ihren Fehler aufmerksam zu machen, indem sie die Problemquelle mit einem darauffolgenden Emoticon ,:D“ verschickt. Janas Reaktion in \#130 zeigt allerdings, dass sie die von Clara eingeleitete Reparaturinitiierung falsch interpretiert hat, da sie die Problemquelle „Gauda“ als Teil eines Kompositums wiederholt und mit dem Emoji signalisiert, dass sie Claras Nachricht nicht richtig einordnen kann. Clara geht allerdings nicht 
weiter darauf ein, dafür führt aber Schreiberin Lili 30 Minuten später in \#131 eine fremdinitiierte Fremdkorrektur durch und verschickt die orthographisch richtige Schreibung „Gouda“ mit einem vorangestellten Asterisk und dem nachgestellten ,zwinkernden Emoji $:$, das man in dem Fall als eine die gesichtsbedrohende Wirkung eines solchen Reparaturtyps abschwächende Ressource interpretieren kann. Eine dreißigminütige Verzögerung zwischen den Nachrichten \#130 und \#131 lässt sich nicht unbedingt mit der Dispräferenz von fremdinitiierten Fremdreparaturen in Verbindung bringen, sondern ist hier vermutlich dadurch bedingt, dass Lili in dieser Zeit nicht an ihrem Smartphone war. Zwei weitere fremdinitiierte Fremdreparaturen weisen ebenso Asteriske in der vorangestellten Position auf. Dabei muss jedoch angemerkt werden, dass zwei davon aus den in 5.1.1.1 erwähnten Gruppenchats stammen, und zwar von den Schreibern, die auch selbstinitiierte Selbstreparaturen mit Asterisken in der gleichen Position kennzeichnen. Daher werden weitere Beispiele benötigt, um festzustellen, ob auch nachgestellte Asteriske fremdinitiierte Fremdreparaturen markieren können.

\subsubsection{Asteriske bei fremdinitiierten Selbstreparaturen}

Mit Asterisken markierte fremdinitiierte Selbstreparaturen kommen in den untersuchten WhatsApp-Daten lediglich zweimal vor. In beiden Fällen liegen Substitutionen vor (vgl. 17 und 18). In (17) wird die von Markus in \#1582 getroffene Wortwahl von Hendrick in Frage gestellt:

(17) aus der Mobile Communication Database 2.0: Auszug aus dem WhatsApp-Gruppenchat \#Qy1Pp

Bei mir auch nix draußen Meine Jacke ist kaputt $A$ ?

Hendrick (Nachricht \#1581 - 22.02.2018 - 17:00)

Beste Ausrede $\Leftrightarrow$

Markus (Nachricht \#1582 - 22.02.2018 - 17:04)

$\Leftrightarrow$

Janus (Nachricht \#1583 - 22.02.2018 - 17:07) 


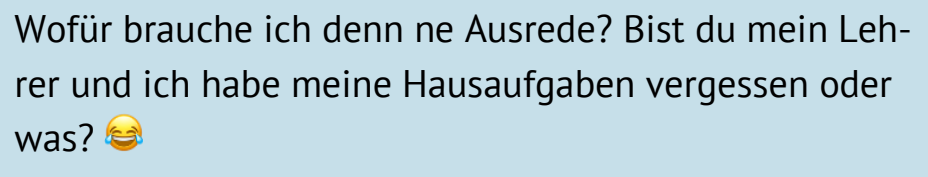

Hendrick (Nachricht \#1584 - 22.02.2018 - 17:12)

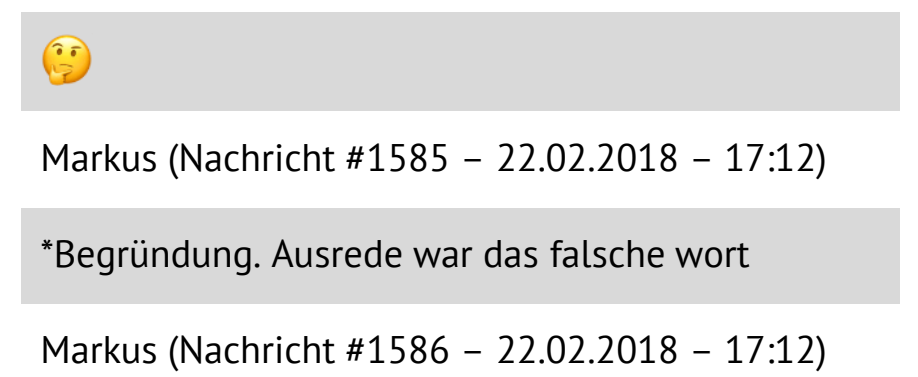

In einem Gruppenchat diskutieren Schreiber, welches Fußballspiel sie sich anschauen wollen und wo das geschehen soll. Dabei äußert sich Hendrick in \#1581 gegen den Vorschlag, ein Spiel draußen anzuschauen, da seine Jacke kaputt sei. Markus bezeichnet in \#1582 Hendricks Argument als eine Ausrede, was jedoch von Hendrick in \#1584 zurückgewiesen wird, indem er Markus darauf hinweist, dass das Wort Ausrede in dem Kontext pragmatisch unpassend sei. Markus geht auf Hendricks Reparaturinitiierung ein, indem er zuerst ein „nachdenkendes“ Emoji 9 verschickt, das möglicherweise seine mentale Auseinandersetzung mit der Problemquelle repräsentieren soll. Anschließend liefert er eine Alternative zum Reparandum, nämlich „Begründung“, mit einem davorstehenden Asterisk, sowie einen Metakommentar, in dem er Hendrick seine unpassende Wortwahl explizit eingesteht. Im Gegensatz zu selbstinitiierten Selbstreparaturen und fremdinitiierten Fremdreparaturen, bei denen die Reparaturinitiierung und -durchführung in der gleichen Nachricht erfolgen, sodass man nicht genau feststellen kann, welcher Komponente einer Reparatursequenz Asteriske zugehören, liefert Nachricht \#1586 einen eindeutigen Hinweis darauf, dass der Asterisk in dem Fall im Kontext einer Reparaturdurchführung eingesetzt wird, um das Reparans zu kennzeichnen.

In der WhatsApp-Kommunikation, in der sowohl schriftbasierte Beiträge als auch Sprachnachrichten zur Verfügung stehen, steht

9 Emojis sowie Emoticons kommen systematisch bei Reparaturinitiierungen und/oder -durchführungen vor (vgl. Beispiele 6,18 und 20). In den untersuchten Daten werden sie allerdings fast nie alleine verwendet, sondern an verbale Initiierungsressourcen gekoppelt bzw. in die Bearbeitung der Problemquelle integriert. Dabei werden Emojis und Emoticons v. a. dazu eingesetzt, um eine die Reparaturinitiierung oder -durchführung enthaltene Nachricht zu modalisieren, um Beziehungsarbeit zu betreiben oder um die gesichtsbedrohende Wirkung einer Fremdreparatur abzuschwächen (zu Funktionen von Emoticons und Emojis vgl. Imo 2015b und Pappert 2017). 
eine entsprechend breite Palette medialer Realisierungsformen der drei Grundkomponenten einer Reparatursequenz (Reparandum Reparaturinitiierung - Reparaturdurchführung) bereit. Alle drei Komponenten einer Reparatursequenz können entweder nur in schriftlicher oder nur in mündlicher Form vorliegen. Da der Schwerpunkt der vorliegenden Arbeit auf der Erforschung graphischer Ressourcen liegt, wurden Reparaturen, die ausschließlich mündlich erfolgen, bei der Analyse nicht mitberücksichtigt. Darüber hinaus steht potentiell noch eine dritte Option zur Verfügung, nämlich, dass verschiedene Komponenten einer Reparatursequenz medial unterschiedlich realisiert werden. Bei einem Beispiel (18) aus der MoCoDa 1.0 liegt die Problemquelle in der Sprachnachricht, wird jedoch schriftlich bearbeitet.

Am Anfang der Dialogsequenz steigen die Schreiberinnen mit einem kleinen Small-Talk ein. Danach fragt Schreiberin B ihre Kommunikationspartnerin nach ihren Plänen für den Tag. Nachdem Schreiberin A diese Frage in Nachricht \#4 beantwortet hat, fragt sie zurück. Schreiberin B reagiert auf diese Frage mit einer Sprachnachricht, deren Inhalt leider nicht miterfasst wurde, und in der sich, wie es sich im späteren Verlauf des Dialogs herausstellt, offensichtlich die ursprüngliche Problemquelle befindet:

(18) aus der Mobile Communication Database 1.0: Auszug aus dem WhatsApp-Dialog \#4311

Wie gehts dirr?? Wir haben voll lane nicht geschrie-

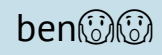

Nachricht \#1 - 24.10.2016 - 11:55:24

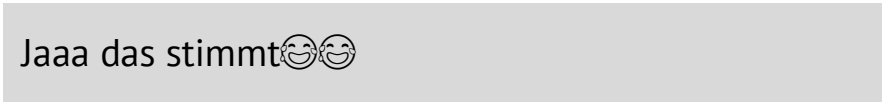

Nachricht \#2 - 24.10.2016 - 12:20:16

Und was machst du sooo heute???

Nachricht \#3 - 24.10.2016 - 12:20:25

Die uni hat angefangen heute Bin in münster

Nachricht \#4 - 24.10.2016 - 12:26:58

Und du??

Nachricht \#5 - 24.10.2016 - 12:27:04 
<Audio weggelassen>

Nachricht \#6 - 24.10.2016 - 12:27:06

Wiee cool ich freuu nichh für dichhh
Nachricht \#7 - 24.10.2016 - 12:31:40
Dann hast du ja jetzt auch ein ticket und kannst
mich besuchen kommen
Nachricht \# 8 - 24.10.2016 - 13:45:08
Ich habe den namen des mädchens nicht gabz ver-
standne wen meinst du?

Nachricht \#9 - 24.10.2016 - 13:45:31

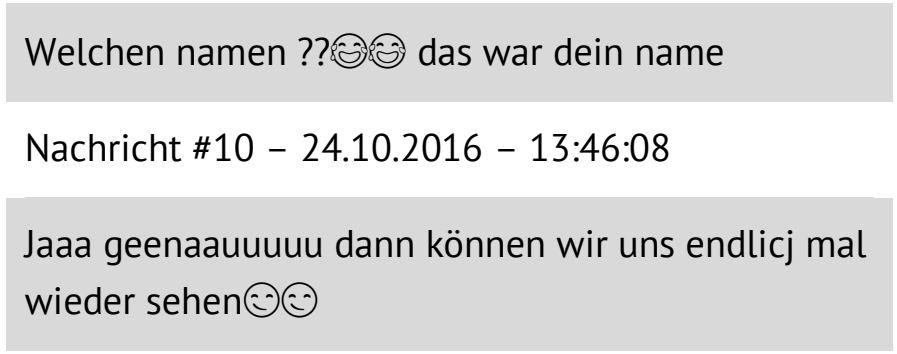

Nachricht \#11 - 24.10.2016 - 14:11:07

Neinn du hast gesagt kennst du "nel" oder sooo

Nachricht \#12 - 24.10.2016 - 14:21:10

Achh sooo *Jimin die asiaten die bei uns auch war

Nachricht \#13 - 24.10.2016 - 14:21:36

Aus den Nachrichten \#7-9 geht hervor, dass Schreiberin B in ihrer Sprachnachricht anscheinend mehrere Themen angesprochen hat: Unter anderem hat sie einen Mädchennamen erwähnt, den Schreiberin A jedoch akustisch nicht verstehen kann, worauf sie ihre Kommunikationspartnerin in Nachricht \#9 explizit hinweist. Damit initiiert sie die erste Reparatur, auf die Schreiberin B in der Nachricht \#10 eingeht. Dabei kann der erste Teil der Nachricht \#10, nämlich „welchen Namen?“, der in Form einer Wiederholung eines Elements der vorangegangenen Nachricht in Kombination mit einem Interrogativpronomen realisiert wird, als Reparaturinitiierung zu Nachricht 
\#9 interpretiert werden (vgl. die Initiierungsformate Teilwiederholung mit Fragewort bei Egbert 2009: 100, bzw. partial repeat of the trouble-source turn with question word bei Schegloff et al. 1977: 368). Im zweiten Teil wird mit „das war dein Name“ die von A initiierte Selbstreparatur durchgeführt, indem B präzisiert, dass sie den Namen von Schreiberin A meinte. In der nächsten Nachricht stellt sich jedoch heraus, dass Schreiberin A einen anderen Mädchennamen, der in der Sprachnachricht anscheinend vorkam, gemeint hat. Sie versucht nun, das entstandene Verständnisproblem zu klären, indem sie B's Interpretationsvorschlag ablehnt und ein in Anführungszeichen eingeschlossenes candidate hearing „"nel"“ liefert. Darauf reagiert Schreiberin B mit dem Erkenntnisprozessmarker (vgl. Imo 2009) ach so, quittiert damit das Verständnis der von A durchgeführten Reparatur und zeigt an, dass sie jetzt verstanden hat, welchen Namen A die ganze Zeit gemeint hat (vgl. Golato/Betz $2008 \mathrm{zu}$ ach so bei third-position repairs). Anschließend führt Schreiberin B eine Selbstreparatur durch, indem sie den Mädchennamen Jimin, gekennzeichnet mit dem vorangestellten Asterisk als Reparaturmarker, verschickt. Danach liefert sie eine zusätzliche Erläuterung, woher sie bzw. die beiden Schreiberinnen diese Person kennen.

Die Reparatur in (18) repräsentiert somit eher einen Ausnahmefall der Verwendung von Asterisken, bei dem ein in der Sprachnachricht vorliegendes Problem des Hörens nicht mündlich, sondern schriftlich bearbeitet wird. Das Aufeinandertreffen medialer Mündlichkeit und medialer Schriftlichkeit innerhalb einer Reparatursequenz ist eine der möglichen Fragestellungen für zukünftige Untersuchungen. Dabei könnten beispielsweise Daten aus der sich gerade im Aufbau befindenden MoCoDa 2.0 herangezogen werden, die eine Möglichkeit bietet, Sprachnachrichten in Form von Transkripten mitzuerfassen.

\subsection{Plus- und Minuszeichen}

Im Dortmunder Chat-Korpus wurden 12 Fälle festgestellt, in denen ein Pluszeichen als Reparaturmarker eingesetzt wird. In den meisten Beispielen wird das Pluszeichen für die Behebung von Tippfehlern im Rahmen einer selbstinitiierten Selbstkorrektur benutzt (vgl. 3.2). Dabei folgen einem Pluszeichen in der Regel ein Buchstabe bzw. ein Interpunktionszeichen, der bzw. das in einen der vorherigen Beiträge desselben Schreibers eingefügt werden soll: 
(19) aus dem Dortmunder Chat-Korpus: Auszug aus Dokument 2221004_ unicum_19-02-2003.xml

705 Shadow die Umstellung von ommer-auf Winterzeit?

706 Shadow +S

In Chat-Ausschnitt (19) vertippt sich Shadow und führt in dem nachfolgenden Beitrag eine selbstinitiierte Selbstkorrektur durch, indem er/sie den fehlenden Buchstaben „S“ postet. Das vorangestellte Pluszeichen deutet darauf hin, dass dieser Buchstabe zum vorherigen Beitrag derselben Person gehört. Die Großschreibung erleichtert dem Leser die Zuordnung des Zeichens in der ursprünglichen Äußerung, nämlich „ommer- auf Winterzeit“. Diese Verwendungsweise von Pluszeichen ähnelt dem Gebrauch des Asterisks in (2), der ebenfalls eine Insertion markiert. Die Frage, ob Pluszeichen und Asteriske als konkurrierende Formen zur Kennzeichnung einer Insertion existiert haben bzw. ob ein Zeichen das andere in dieser Funktion abgelöst hat, muss vorerst offenbleiben.

Während in der Forschungsliteratur Beispiele erwähnt werden, in denen Pluszeichen ausschließlich im Kontext selbstinitiierter Selbstkorrekturen vorkommen (vgl. Abschnitt 3.2), zeigen die untersuchten Chat-Daten, dass Pluszeichen auch eine Insertion bei einer fremdinitiierten Fremdreparatur markieren können, wie in (20):

(20) aus dem Dortmunder Chat-Korpus: Auszug aus Dokument 2221010 _ unicum_01-07-2003.xml

$\begin{array}{lll}386 & \text { dieNachbarin } & \text { bye allemale } \\ 387 & \text { Luna84 } & \begin{array}{l}\text { beibei marcie und pööörlie ! ( los, geh lernen !!! } \\ (:)\end{array} \\ 388 & \text { rebell } & \text { cu nachbarin } \\ 389 & \text { dieNachbarin } & + \text { die! } \\ 390 & \text { dieNachbarin } & \text {;) }\end{array}$

Schreiberin dieNachbarin initiiert in Zeile 386 eine Abschiedssequenz, auf die andere Chatter reagieren, unter anderen auch rebell, der/die in die Verabschiedungsfloskel den Nickname des Gegenübers miteinbezieht, diesen jedoch nicht vollständig vewendet, nämlich ohne den definiten Artikel die, worauf er/sie direkt von $d i$ eNachbarin aufmerksam gemacht wird. dieNachbarin führt eine fremdinitiierte Fremdreparatur durch, indem sie den fehlenden Artikel mit einem Pluszeichen und einem nachgestellten Ausrufezeichen postet, das ihrer Reparatur zusätzlichen Nachdruck verleiht. Diese Handlung belegt die wichtige kommunikative Relevanz von 
Nicknames in der CMC (vgl. Beispiele 5a und 15). Um jedoch die durch die Fremdreparatur entstandene gesichtsbedrohende Wirkung (vgl. Schegloff et al. 1977; Hutchby/Wooffitt 1998: 68) zu mildern, postet dieNachbarin anschließend ein ,zwinkerndes' Emoticon.

Während Pluszeichen zur Kennzeichnung eines inserierenden Elements benutzt werden, zeigen 12 im Unicum-Subkorpus ${ }^{10}$ vorgefundene Minuszeichen ein $\mathrm{zu}$ tilgendes Zeichen an. Dabei wird i. d. R. nach einem Beitrag mit dem Reparandum eine Kombination aus einem Minuszeichen und einem zu tilgenden Buchstaben verschickt, analog zu (5a). Darüber hinaus findet sich in (21) eine weitere Strategie, bei der das Reparandum und die oben genannte Kombination im selben Beitrag erscheinen:

(21) aus dem Dortmunder Chat-Korpus: Auszug aus Dokument 2221010_ unicum_01-07-2003.xml

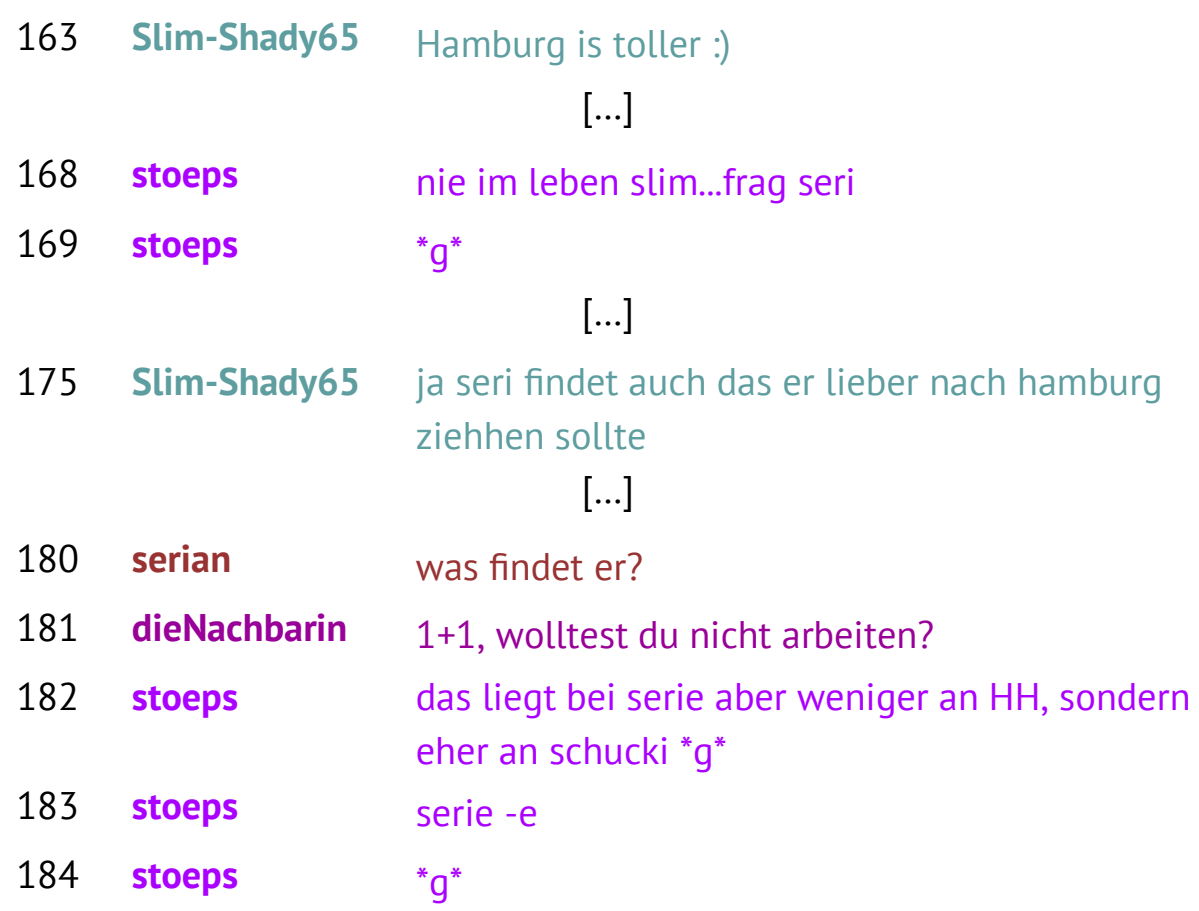

Im Laufe einer Diskussion darüber, wo man am besten hinziehen sollte (nach Baden-Württemberg oder nach Hamburg), plädiert Slim-Shady65 für Hamburg und wird von stoeps auf serian verwiesen, der vermutlich negative Erfahrungen mit Hamburg gemacht haben soll. In Zeile 182 erläutert stoeps näher, dass diese Erfahrungen nichts mit der Stadt selbst zu tun haben, sondern eher persönliche

10 Das einzige Minuszeichen in der MoCoDa 2.0 stammt aus einem WhatsAppDialog auf Englisch, daher wird es aus der Analyse ausgeschlossen. 
Gründe haben. Dabei vertippt sie sich beim Nickname und gibt anstatt der Kurzform seri das Wort „serie“ ein. In der nächsten Zeile korrigiert sie ihren Fehler, indem sie zuerst das Reparandum postet und danach das „e“ mit einem Minuszeichen davor, um anzuzeigen, dass dieses getilgt werden soll.

Während ein Pluszeichen eine Insertion und ein Minuszeichen eine Tilgung abbildet, kann man mit Hilfe der Kombination aus beiden eine Substitution als Reparaturoperation erzeugen, wie in (22). Es liegen insgesamt zwei solche Fälle im Unicum-Korpus vor, die in der Tabelle (s. o. auf S. 54) mit Einträgen (+2) sowohl bei Minuszeichen als auch bei Pluszeichen dokumentiert sind:

(22) aus dem Dortmunder Chat-Korpus: Auszug aus Dokument 2221005 unicum_20-02-2003.xml

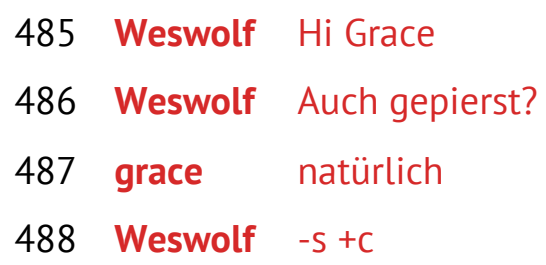

Die selbstinitiierte Selbstkorrektur von Weswolf bezieht sich auf das Reparandum „gepierst“. Die Korrektur zeigt an, dass man in „gepierst" das $s$ tilgen und an seine Stelle ein $c$ einfügen soll, damit die richtige Form gepierct ensteht.

\subsection{Gleichheitszeichen}

Im Gegensatz zu Plus- und Minuszeichen kommen Gleichheitszeichen in allen drei Korpora vor. Dabei sind sie im Dortmunder ChatKorpus öfter als in den beiden WhatsApp-Korpora vertreten (10 vs. 2 Fälle). Darüber hinaus weisen Gleichheitszeichen im UnicumKorpus eine breitere Funktionalität auf: Während sie in der MoCoDa 1.0 und 2.0 lediglich in Fremdinitiierungen vorkommen (vgl. Beispiel 24), werden Gleichheitszeichen in den Daten aus dem IRCChat außerdem auch bei selbstinitiierten Reparaturen - v. a. bei zu korrigierenden Tippfehlern sowie bei unklaren Referenzen -eingesetzt. Eine selbstinitiierte Selbstkorrektur eines Elementes des Zuschreibungsturns (vgl. Storrer 2001) liegt in (23) vor: 
(23) aus dem Dortmunder Chat-Korpus: Auszug aus Dokument 2221006 unicum_21-02-2003_1.xml

\begin{tabular}{|c|c|c|}
\hline 192 & TomcatMJ & "auf bruam raufkletter um nich angeleint zu werden" \\
\hline 193 & Lantonie & $\begin{array}{l}\text { http://www.cu-here.de/showchat.php3?id=69\&off- } \\
\text { set=159 }\end{array}$ \\
\hline 194 & Shadok & tag quaki \\
\hline 195 & zora & erste! :) \\
\hline 196 & & hmmm quaki sucht ein $\mathrm{m}$ \\
\hline 197 & TomcatMJ & ${ }^{*} g^{*}$ bruam=baum \\
\hline
\end{tabular}

Im Zuschreibungsturn in Zeile 192 produziert TomcatMJ das Wort „bruam“ anstelle von baum. Dieser Vertipper wird von ihm in Zeile 197 korrigiert: Dafür nutzt er die Funktion von =, zwei Elemente gleich zu setzen. Er platziert das Reparandum und das Reparans jeweils links bzw. rechts von einem Gleichheitszeichen und signalisiert damit, dass „baum“ der Problemquelle „bruam“ aus Zeile 192 entspricht und diese daher ersetzen soll. Hinsichtlich der Reparaturoperation zielt diese Korrektur also auf eine Substitution ab.

Darüber hinaus tauchen Gleichheitszeichen sowohl im Dortmunder Chat-Korpus als auch in den beiden MoCoDa-Korpora bei Fremdinitiierungssequenzen auf, in denen ein candidate understanding angeboten wird, wie in (24). In einem Dialog zwischen zwei Freundinnen deutet B ganz am Anfang an, dass sie ein Beziehungsproblem mit ihrem Freund Alex hat, über das sie jedoch erst am Freitag erzählen will. Nach einer Besprechung des bevorstehenden Treffens, das von Freitag auf Samstag verlegt wird, kehrt B dennoch schon in diesem Dialog zu ihrem Beziehungsproblem zurück. Dabei beschwert sich B bei A darüber, dass ihr Freund sich komisch verhält, weil er immer öfter meint, dass er keine Lust auf eine Beziehung mit ihr hat. Anschließend liefert B auch den Grund dafür, wieso sie die Besprechung ihres Beziehungsproblems ursprünglich auf Freitag verschieben wollte, nämlich, weil sie sich gerade bei ihrem Freund aufhält:

(24) aus der Mobile Communication Database 1.0: Auszug aus dem WhatsApp-Dialog \#4106

Alex sagt ungefähr jeden zweiten Tag er hätte kein Bock mehr aber ist dann voll normal und keine Ahnung will Urlaub buchen und so voll komisch

Nachricht \#11 - 13.06.2016 - 19:23:05 


\section{Er meint ich sei knatschig}

Nachricht \#12 - 13.06.2016 - 19:23:05

Marianne ich bin auch gerade bei dem (20)

Nachricht \#13 - 13.06.2016 - 19:23:05

Oh

Nachricht \#14 - 13.06.2016 - 19:24:05

Woher soll ich das wissen haha

Nachricht \#15 - 13.06.2016 - 19:24:05

Hahaha

Nachricht \#16 - 13.06.2016 - 19:24:05

Ja kein bock mehr $=$ Schlus machen $?$

Nachricht \#17 - 13.06.2016 - 19:25:05

Sonst hätte ich dir das jetzt

ausführlich erzählt hahs

Nachricht \#18 - 13.06.2016 - 19:25:05

Ihr hattet doch in letzter Zeit kaum Stress..

Nachricht \#19 - 13.06.2016 - 19:25:05

Jz bin ich neugierig

Nachricht \#20 - 13.06.2016 - 19:25:05

Ja keine Ahnung kann ich mir nicht vorstellen weil der dann andererseits wieder so weit im Voraus alles planen will

Nachricht \#21 - 13.06.2016 - 19:26:05

Die von B in \#11 geschilderte Beziehungskrise wird von A aufgegriffen, indem sie in \#17 ihr Bedeutungsverstehensproblem mit dem Ausdruck „kein bock mehr“ zum Ausdruck bringt. Dafür liefert sie 
das candidate understanding „Schluss machen“, das sie mit Hilfe eines Gleichheitszeichens mit der von ihr wiederholten Problemquelle in Relation setzt. Dadurch werden potentielle Schwierigkeiten bei der Erkennung der Problemquelle, die fünf Nachrichten zurückliegt, vermieden. Die Reaktion auf diese Fremdinitiierung erfolgt in \#21. Dabei lässt sich das nachrichteninitiale „Ja" nicht als eine Bestätigung in Bezug auf die Initiierungsnachricht, sondern eher entweder als Rückversicherungssignal oder als eine Bestätigung A’s Annahme in \#19 interpretieren. Implizit weist B das von A vorgelegte candidate understanding „Schluss machen“ zurück, indem sie den Wunsch ihres Freundes, etwas zusammen zu planen, als Argument dafür anführt, dass ihre Beziehung weiter bestehen bleibt.

Dadurch, dass sowohl in (23) als auch in (24) das Reparandum wiederaufgenommen wird und entweder mit dem Reparans oder einem candidate understanding gleichgesetzt wird, erlauben Gleichheitszeichen als graphische Reparaturressourcen, eine zeitlich und sequentiell zurückliegende Problemquelle effizient zu bearbeiten.

\section{Fazit und Ausblick}

In der vorliegenden Untersuchung wurde gezeigt, dass sowohl in der Kommunikation via IRC-Chat als auch über WhatsApp graphische Ressourcen bei der Reparaturinitiierung bzw. -durchführung eingesetzt werden. Dabei hat sich herausgestellt, dass die meisten graphischen Mittel zwar bei selbstinitiierten Selbstreparaturen auftauchen (was vermutlich durch die Präferenz und entsprechend hohe Zahl dieses Reparaturtyps bedingt ist (vgl. Schegloff et al. 1977)), dennoch finden sich einige Einzelfälle bei fremdinitiierten Selbst- sowie Fremdreparaturen.

Graphische Ressourcen können dabei unterschiedliche Funktionen innerhalb einer Reparatursequenz ausüben. Zum einen können sie das Reparans markieren und dabei die durchzuführende Reparaturoperation offenlegen. In den Untersuchungskorpora wurden beispielsweise Asteriske konsequent bei Substitutionen und Insertionen eingesetzt. Weitere graphische Zeichen, die eine Reparaturoperation mitkodieren, sind u. a. Minuszeichen (= Tilgung), Pluszeichen (= Insertion) oder Gleichheitszeichen (= Substitution). Des Weiteren dienen Gleichheitszeichen dazu, das Reparandum und das Reparans in Relation zueinander zu setzen, sodass die zu ersetzende Problemquelle explizit thematisiert wird, wodurch v. a. weiter zurückliegende Reparanda erfolgreich bearbeitet werden können.

Neben der Darstellung der Form und Funktion graphischer Ressourcen bei einer Reparatur wurde in dieser Arbeit ein Vergleich in 
Bezug auf die Kommunikationsform (IRC-Chat vs. WhatsApp) angestrebt. Dieser zeigt v. a., dass sich Asteriske als Reparaturmarker im Gegensatz zu Plus- und Minuszeichen durchgesetzt haben. Darüber hinaus kommen Asteriske in den WhatsApp-Dialogen aus dem Jahr 2018 nicht nur immer noch (und im Vergleich zu den Daten aus der MoCoDa 1.0 - also in WhatsApp-Dialogen zwischen 2013 und 2017 - sogar etwas häufiger) vor, sondern weisen außerdem ein breiteres Funktionsspektrum bei unterschiedlichen Reparaturen, Reparandatypen sowie Reparaturoperationen auf.

\section{Literatur}

Beißwenger, Michael (2010): Chattern unter die Finger geschaut: Formulieren und Revidieren bei der schriftlichen Verbalisierung in synchroner internetbasierter Kommunikation. In: Ágel, Vilmos/Hennig, Mathilde (Hg.): Nähe und Distanz im Kontext variationslinguistischer Forschung. Berlin, New York: de Gruyter, 247-294.

Cherny, Lynn (1999): Conversation and community: Chat in a virtual world. Stanford, California: CSLI Publications.

Christopherson, Laura (2013): OMG! l2spell online: The creative vocabulary of cyberlanguage $s\left(\sim_{-}^{1}\right)--b$. PhD thesis, University of North Carolina. URL: https://cdr.lib.unc.edu/indexablecontent/ uuid:dff2f035-a149-4da5-9e7f-6f09c32180d6.

Collister, Lauren (2008): Virtual Discourse Structure: An Analysis of Conversation in World of Warcraft. Master thesis, University of Pittsburgh. URL: http://d-scholarship.pitt.edu/7992/1/collistermathesis2008-virtual_discourse_structure_1.pdf.

Collister, Lauren (2011): *-repair in Online Discourse. In: Journal of Pragmatics 43 (3), 918-921. DOI: https://doi.org/10.1016/j.pragma. 2010.09.025.

Collister, Lauren (2012): The discourse deictics ${ }^{\wedge}$ and <-- in a World of Warcraft community. In: Discourse, Context, and Media 1 (1), 9-19. DOI: https://doi.org/10.1016/j.dcm.2012.05.002.

Couper-Kuhlen, Elizabeth/Selting, Margret (2018): Interactional Linguistics. Studying Language in Social Interaction. Cambridge: Cambridge University Press.

Darics, Erika (2012): Instant Messaging in Work-Based Virtual Teams: the Analysis of Non-Verbal Communication used for the Contextualisation of Transactional and Relational Communicative Goals. PhD diss., Loughborough University. URL: http://erika.darics.co.uk/download/darics_phd.pdf. 
Drew, Paul (1997): 'Open' Class Repair Initiators in Response to Sequential Sources of Troubles in Conversation. In:Journal of Pragmatics 28, 69-101.

Egbert, Maria (2009): Der Reparatur-Mechanismus in deutschen Gesprächen. Mannheim: Verlag für Gesprächsforschung. URL: http://www.verlag-gespraechsforschung.de/2009/pdf/reparaturen.pdf.

Golato, Andrea/Betz, Emma (2008): German ach und achso in repair uptake: Resources to sustain or remove epistemic asymmetry. In: Zeitschrift für Sprachwissenschaft 27 (1), 7-37.

Goloshubina, Olesya (2016): Metayazykovaya refleksiya v internetkommunikatsii (na primere rechevogo zhanra "razgovor v messendzhere") [Metalinguistic Reflection in Internet Communication (Examples of the Speech Genre "Conversation in the Messenger")]. In: Nauka o cheloveke: gumanitarnye issledovaniya 3 (25), 59-65. DOI: 10.17238/issn1998-5320.2016.25.59.

Gysin, Daniel (2015): Höflichkeit und Konfliktbewältigung in der Online-Kommunikation Jugendlicher. Dissertation, Pädagogische Hochschule Karlsruhe. URL: http://nbn-resolving.de/urn:nbn:de: bsz:751-opus4-608.

Henn-Memmesheimer, Beate/Eggers, Ernst (2010): Inszenierung, Etablierung und Auflösung: Karriere einer grammatischen Konstruktion im Chat zwischen 2000 und 2010. In: Networx 57. URL: http://www.mediensprache.net/networx/networx-57.pdf. DOI: $10.15488 / 2948$.

Hutchby, Ian/Wooffitt, Robin (1998): Conversation Analysis Principles, Practices and Applications. Cambridge: Polity Press.

Imo, Wolfgang (2009): Konstruktion oder Funktion? Erkenntnisprozessmarker (change-of-state-token) im Deutschen. In: Günthner, Susanne/Bücker, Jörg (Hg.): Grammatik im Gespräch. Berlin: de Gruyter, 57-86.

Imo, Wolfgang (2015a): Nachträge im Spannungsfeld von Medialität, Situation und interaktionaler Funktion. In: Vinckel-Roisin, Hélène (Hg.): Das Nachfeld im Deutschen: Theorie und Empirie. Berlin: de Gruyter, 231-253.

Imo, Wolfgang (2015b): Vom ikonischen über einen indexikalischen zu einem symbolischen Ausdruck? Eine konstruktionsgrammatische Analyse des Emoticons :-). In: Bücker, Jörg/Günthner, Susanne/Imo, Wolfgang (Hg.): Konstruktionsgrammatik V: Konstruktionen im Spannungsfeld aus sequenziellen Mustern, kommunikativen Gattungen und Textsorten. Tübingen: Stauffenburg, $133-162$.

Jacobs, Jennifer Baker/Garcia, Angela Cora (2013): Repair in chat room interaction. In: Herring, Susan/Stein, Dieter/Virtanen, Tuija (Hg.): Pragmatics of Computer-Mediated Communication. Berlin: 
de Gruyter, 565-588. DOI: https://doi.org/10.1515/9783110214468 .565 .

Kim, Soh Youn (2006): The effects of computer-mediated interaction in L2 vocabulary learning. Master thesis, Iowa State University. URL: https://lib.dr.iastate.edu/cgi/viewcontent.cgi?article=17118\& context=rtd.

Kitzinger, Celia (2013): Repair. In: Sidnell, Jack/Stivers, Tanya (Hg.): The Handbook of Conversation Analysis. Oxford: Wiley-Blackwell, 229-256.

Lemnitzer, Lothar/Naumann, Karin (2001): "Auf Wiederlesen! — das schriftlich verfaßte Unterrichtsgespräch in der computervermittelten Kommunikation. Bericht von einem virtuellen Seminar. In: Beißwenger, Michael (Hg.): Chat-Kommunikation. Sprache, Interaktion, Sozialität und Identität in synchroner computervermittelter Kommunikation. Stuttgart: ibidem, 469-491.

Meredith, Joanne/Stokoe, Elizabeth (2013): Repair: Comparing Facebook 'chat' with spoken interaction. In: Discourse \& Communication 8 (2), 181-207. DOI: http://journals.sagepub.com/doi/pdf /10.1177/1750481313510815.

Mostovaia, Irina (i. V.): Reparaturmechanismen in der interaktionellen informellen Schriftichkeit. Eine kontrastive empirische Untersuchung der Reparaturen in der deutschen und russischen SMS- und WhatsApp-Kommunikation. Universität Hamburg.

Pappert, Steffen (2017): Zu kommunikativen Funktionen von Emojis in der WhatsApp-Kommunikation. In: Beißwenger, Michael (Hg.): Empirische Erforschung internetbasierter Kommunikation. Berlin: de Gruyter, 175-211.

Pfeiffer, Martin (2015): Selbstreparaturen im Deutschen. Syntaktische und interaktionale Analysen. Berlin, Boston: de Gruyter.

Pfeiffer, Martin (2017): Über die Funktion der Reparaturmarker im Deutschen. In: Blühdorn, Hardarik/Deppermann, Arnulf/Helmer, Henrike/Spranz-Fogasy, Thomas (Hg.): Diskursmarker im Deutschen. Reflexionen und Analysen. Göttingen: Verlag für Gesprächsforschung, 259-283.

Piperski, Aleksandr/Somin, Anton (2014): Prednamerennoe zacherkivanie $\mathrm{v}$ internete $\mathrm{i}$ ego analogi $\mathrm{v}$ razlichnykh kommunikativnykh sredakh [Deliberate Strikethrough on the Web and its Counterparts in Different Communicative Environments]. In: Vestnik Sankt-Peterburgskogo gosudarstvennogo universiteta tekhnologii i dizaina 3, 63-69. URL: http://t1p.de/ksyc.

Schegloff, Emanuel A./Jefferson, Gail/Sacks, Harvey (1977): The preference for self-correction in the organization of repair in conversation. In: Language 53 (2), 361-382.

Schegloff, Emanuel A. (1997): Practices and Actions: Boundary Cases of Other-Initiated Repair. In: Discourse Processes 23 (3), 499-545. 
Schegloff, Emanuel A. (2013): Ten operations in self-initiated, sameturn repair. In: Hayashi, Makoto/Raymond, Geoffrey/Sidnell, Jack (Hg.): Conversational Repair and Human Understanding. Cambridge: Cambridge University Press, 41-70.

Scheuplein, Tanja (2012): Code-Sreitching-Ereignisse in der Chatkommunikation: Wie im Chat mit Sprachwechselphänomenen umgegangen wird. Stuttgart: ibidem.

Schönfeldt, Juliane/Golato, Andrea (2003): Repair in Chats: A Conversation Analytic Approach. In: Research on Language and Social Interaction 36 (3), 241-284. DOI: 10.1207/S15327973RLSI3 603_02.

Schrader, Frederik (2010): Sprachverwendung im Game-Chat. Eine Korpusanalyse in Abgrenzung zur konventionellen Chat-Kommunikation und mündlichen Konzeption. Magisterarbeit. Hamburg: Diplomica Verlag.

Stæhr, Andreas (2014): Social media and everyday language use among Copenhagen youth. $\mathrm{PhD}$ thesis, Københavns Universitet. URL: http://curis.ku.dk/ws/files/122495013/Ph.d._2014_Staehr.p df.

Storrer, Angelika (2001): Getippte Gespräche oder dialogische Texte? Zur kommunikationstheoretischen Einordnung der ChatKommunikation. In: Lehr, Andrea/Kammerer, Matthias/Konerding, Klaus-Peter/Storrer, Angelika/Thimm, Caja/Wolski, Werner (Hg.): Sprache im Alltag. Beiträge zu neuen Perspektiven in der Linguistik. Berlin, New York: de Gruyter, 439-465.

Stukenbrock, Anja (2013): Sprachliche Interaktion. In: Auer, Peter (Hg.): Sprachwissenschaft. Stuttgart: Metzler, 217-260.

Vinagre, Margarita/Muñoz, Beatriz (2011): Computer-mediated corrective feedback and language accuracy in telecollaborative exchanges. In: Language Learning $\mathcal{E}$ Technology 15 (1), 72-103. URL: https://scholarspace.manoa.hawaii.edu/bitstream/10125/44 238/1/15_01_vinagremunoz.pdf. 